\title{
Random metric spaces and universality
}

\author{
A. M. Vershik*
}

\begin{abstract}
We define the notion of a random metric space and prove that with probability one such a space is isometric to the Urysohn universal metric space. The main technique is the study of universal and random distance matrices; we relate the properties of metric (in particular, universal) spaces to the properties of distance matrices. We give examples of other categories in which the randomness and universality coincide (graphs, etc.).
\end{abstract}

\section{CONTENTS}

1. Introduction. Relation between randomness and universality.

2. The cone of distance matrices and its geometry. Random distance matrices.

3. Universal distance matrices and the Urysohn space. Urysohnness criterion.

4. The main theorem: universality of almost all distance matrices.

5. Matrix distributions as a complete invariant of metric triples and a generalization of Kolmogorov's problem on extension of measures.

Appendix. Examples of categories where universality and randomness coincide. The universal and random Radó-Erdös-Renyi graph, etc.

\section{Introduction. Relation between randomness and universality}

Random objects in various concrete, relatively simple algebraic and geometric categories (random graphs, random polyhedra, etc.) have been studied for a long time; however, as was already mentioned in [9], only recently such studies started to assume systematic character; one may conjecture that in the near future this direction will become one of the most actual research fields. This is motivated by numerous applications of stochastic models, in particular in the field theory and statistical physics, as well as by needs of mathematics itself, for example in the representation theory, number theory, theory of dynamical systems, and the probability theory itself.

*St. Petersburg Department of Steklov Institute of Mathematics, 27 Fontanka, 191011 St. Petersburg, Russia. Partially supported by the RFBR grant 02-01-00093 and the President of Russian Federation grant for support of leading scientific schools 2251.2003.1. 
The classical probability theory and theory of random processes dealt and deal mainly with scalar or vector random variables, maybe infinite-dimensional. But the foundations of probability theory, which were formulated in the famous work by A. N. Kolmogorov on the basis of general measure theory, of course, make it possible to develop the theory of random objects in arbitrary categories and formulate laws of large numbers and limit theorems that are quite far from the classical ones. For example, we may speak of the limit distribution of partitions of positive integers and Young diagrams, the "law of large representations" for random representations in series of classical groups (see papers on the asymptotic representation theory, e.g., in [26]), or the "laws of large metric spaces" (this will be done in this paper), etc. It is interesting that the technical part of the corresponding considerations uses rather classical probabilistic ideas and terms, but requires solving nontraditional problems. It is equally important that these problems lead to new remarkable distributions, which did not arise earlier (for example, the Airy distribution, the Poisson-Dirichlet distribution, the Lebesgue measure in the space of subordinators, etc., which arose in the theory of random partitions, Young diagrams, or spectra of random matrices).

Some time ago the author became interested in the following question: what is a random metric space, and what can it look like if this question makes any sense. This question arose in connection with some problems of measure theory and ergodic theory (the study of metrics related to classification of filtrations, i.e., decreasing sequences of $\sigma$-algebras) from one hand, and with the study of the remarkable Urysohn universal metric space (see below), which had been undeservedly forgotten and which manifestly pretended to play the central role in such considerations, from the other hand. The main result of this paper and the recent publications [6, 13 is that the Urysohn space is not only universal, but also generic in the topological (categorical) sense, and, which is most important, it is "random" in the following sense: "sufficiently random" finite metric spaces converge to the Urysohn space with probability one.

Comparing with a simpler situation, namely with the theory of random graphs, developed in the 60s, where the universal graph in the sense of Radó turns out, in a trivial way, to be also random in the Erdös-Rényi sense (and, in fact, in much stronger sense - see Section 5), allows one to believe that the coincidence of universality and randomness is not random and occurs in many categories. A brief survey of categories where such a coincidence takes place is given in Appendix and will be considered in more detail elsewhere.

There are several fields related to the problems discussed in this paper; we will mention them only briefly and in connection with concrete problems. First, this is the theory of random matrices, since we consider random distance matrices. A special class of such matrices - matrix distributions - are complete invariants of metric triples (or Gromov triples, or $\mathrm{mm}$-spaces), i.e., Polish spaces with Borel probability measure, regarded up to measure-preserving isometries. A matrix distribution is in turn the distribution of a random distance matrix, which is invariant and ergodic under the action of the group of simultaneous permutations of rows and columns and satisfies a certain technical condition (the simplicity condition, see Section 5); these relations and arising new problems will be mentioned below. In particular, it is especially interesting to study the limit spectrum of the random distance matrix, or, in other 
words, the random metric on the set of positive integers. Second, the universality problems under consideration, as far as we deal with countable spaces, are related to logic, namely to the theory of models (see, e.g., [28]), where one considers the universality (categoricity of a model) for different theories, and in particular for graph theory. For example, there is a striking relation between $0-1$ laws in the theory of first-order languages (see [34, 29, 31, 30, 16, 36]) and similar Kolmogorov-type laws in the theory of random processes. Another parallel with the classical probability theory is related to Kolmogorov's theorem on extension of a cylinder measure to a countably additive measure in the infinite product of spaces; we consider its nonlinear analog: the problem of extending a finitely additive measure defined on the set algebra generated by the family of balls in a metric space centered at the points of a countable everywhere dense system; it turns out that such an extension exists not for all spaces (see Section $5)$.

Let us turn to a more detailed description of the main results and contents of the paper. We begin with a description of the class (category) of equipped metric spaces, i.e., complete separable (= Polish) metric spaces with a distinguished countable wellordered everywhere dense subset. We identify an equipped Polish space with the matrix formed by the distances between the points of the distinguished subset; this matrix is in a certain sense the set of structural constants of the metric space. Such a viewpoint allows us to introduce a topology, define measures, etc., on the space (category) of equipped metric spaces.

In the second section we study the geometry of the cone of distance matrices and the convex set consisting of the vectors formed by the distances from the points of a given finite metric space to a new point attached to this metric space. We define the procedure of random growth of finite metric spaces and give a precise statement of the main problem. The machinery of distance matrices is convenient for studying some properties of metric spaces; for example, in the recent paper [27], it is shown how one can find the Čech homologies of a metric space, given the distance matrix of this space. Recall that in the same time the classification of Polish (noncompact) spaces up to isometry is a "wild" problem (see [7]). The main problem is to define the notion of random metric space and to study the relation between randomness and universality. Note that every metric triple (= Polish space with a nondegenerate Borel probability measure) naturally generates a random distance matrix. Namely, this is the random matrix of the distances between the points of countable (dense) subsets of a realization of the sequence of i.i.d. points of the space distributed according to this measure (see Appendix and [4, 5, 6]). We have the following important question: what invariants of the metric space are contained in the spectrum of these random symmetric matrices; only preliminary experiments related to this question are carried out (see [38]). Recall that the distribution of these matrices (the matrix distribution) is a complete invariant of the metric triple $([4,5,8])$; hence the old question "whether one can hear the form of the drum" (i.e., recover a Riemannian metric from the spectrum of the Laplacian) takes a new form: whether one can recover a metric triple up to volume-preserving isometry from the spectrum of the random matrices of the distances between independently chosen random points of the space. Although the answer to the latter question in the general case is most probably negative (as in the case of the former question), the 
random spectrum undoubtedly contains information on many invariants. This question needs a detailed study.

In the third section we recall the definition of the universal space Iso $(\mathcal{U})$, introduced by P. S. Urysohn in 1924 in the papers [2, 1], which were published already after his tragic death. We introduce the notion of a universal distance matrix and then prove that such and only such matrices can be the distance matrices of countable everywhere dense subsets of the Urysohn space. This "urysohnness" criterion was also given in [6] and 13]; its proof generalizes and simplifies the proof of the main assertions of the original paper by P. S. Urysohn on the existence and uniqueness of the universal Polish space. Our criterion implies immediately that universal matrices form an everywhere dense set in the cone of distance matrices and thus implies the "genericity" of the Urysohn space within the chosen model, i.e., in the sense of the topology of the cone $\mathcal{R}$. It follows in turn that with respect to a generic probability measure on the cone of distance matrices, almost all matrices are universal and thus with probability one determine spaces isometric to the Urysohn space. However, this assertion does not allow us to find at least one such measure explicitly; concrete examples are given in the next section. We give a survey of known properties of the space $\mathcal{U}$.

In the fourth section we prove that the procedure defined in the second section gives explicit examples of measures on the cone of distance matrices with respect to which almost all distance matrices are universal. Random distance matrices governed by these distributions are the best, as far as allowed by the triangle inequality, simulation of random matrices with independent entries. This gives another justification of the thesis that the random metric space is universal.

In the fifth section we reproduce main facts on the classification of metric triples up to measure-preserving isometry, which are described in detail in [6, 8]. We state a theorem on the matrix distribution as a complete invariant of a metric triple and on the reconstruction of the metric triple from this invariant and give a sketch of the proof. This theorem allows us to study invariants of a metric space with measure using only the matrix distribution. The following new effect is of particular interest: in metric spaces (for example, in the Urysohn space) there exist sequences that are uniformly distributed with respect to a certain finitely additive measure, while there exists no countably additive measure with respect to which they would be uniformly distributed. Such examples are given by the same construction from Section 3.

The thesis on coincidence of universality and randomness has a much wider domain of applicability; in Appendix we recall the combinatorial analog of our problem the relation between random and universal graphs, as well as give other examples. Note that the papers [14, 15, 16] on the universal graph appeared almost 40 years later than Urysohn's paper, but it was apparently unknown to their authors. The example of graphs is a quite special case of our scheme; namely, the universal graph is the universal metric space in the class of metrics assuming only the values $0,1,2$ (hence in this case the triangle inequality is automatically satisfied). But a deeper analogy between both theories is not only in the same relation between universality and randomness, but in an analogy between the group of isometries of the Urysohn space and the group of automorphisms of the universal graph; these poorly studied groups are of great interest as universal, in one or another sense, infinite-dimensional 
groups. The group of isometries of the Urysohn rational universal space, which we will mention only briefly, takes an intermediate position between these two examples. The groups of symmetries of universal spaces in various categories are of special interest from the purely algebraic point of view as well as for the whole theory we develop.

The author is grateful to Erwin Schrödinger Institut (Vienna) and Max-PlanckInstitute (Bonn) for offering him the possibility to work. He is also grateful to E. Gordon, P. Cameron, J. Baldwin, Y. Benjamini, B. Granovsky, A. Bovykin, and V. Uspensky for useful references obtained from them at various times. A part of the material of the paper is made up from the lectures given by the author in Vienna University (Austria, October 2002), Santiago University (Chile, December 2002), Les Houches Winter Physics School (France, March 2003), and Haifa Technion (Israel, June 2003). The Ph.D. student U. Haboeck helped me to process the texts of Vienna lectures. The author also thanks the group of physicists (O. Bohigos et al.) from Université Orsay (Paris-IX), who became interested in these subjects after the author's lecture in Oberwolfach and made very useful numerical experiments with spectra of distance matrices for simplest manifolds.

\section{The cone $\mathcal{R}$ of distance matrices and its ge- ometry. Random distance matrices}

\subsection{Definitions}

Consider the following set of infinite real matrices:

$$
\mathcal{R}=\left\{\left\{r_{i, j}\right\}_{i, j=1}^{\infty}: r_{i, i}=0, r_{i, j} \geq 0, r_{i, j}=r_{j, i}, r_{i, k}+r_{k, j} \geq r_{i, j} \text { for } i, j, k=1,2, \ldots\right\} .
$$

Elements of the set $\mathcal{R}$ will be called distance matrices; note that zeros outside the main diagonal are allowed. Every distance matrix determines a semimetric on the set of positive integers $\mathbf{N}$. A matrix from $\mathcal{R}$ is called a proper distance matrix and determines a metric on $\mathbf{N}$ if it has no zeros outside the main diagonal. The set of all distance matrices is a convex cone in the vector space of infinite real matrices $\operatorname{Mat}_{\mathbf{N}}(\mathbf{R})=\mathbf{R}^{\mathbf{N}^{2}}$; this cone is weakly closed in the ordinary weak topology in the space of infinite real matrices. We will call it the cone of distance matrices. The subset of proper distance matrices is an open weakly dense subcone in $\mathcal{R}$. If $r$ is a proper distance matrix, then the completion of the metric space $(\mathbf{N}, r)$ is a complete separable metric (= Polish) space $\left(X_{r}, \rho_{r}\right)$ equipped with a countable everywhere dense well-ordered subset $\left\{x_{i}\right\}_{i=1}^{\infty}$, which is the image of the set of positive integers in the completion. An arbitrary distance matrix (with possible zeros outside the main diagonal) determines a semimetric on the set of positive integers; in this case, before constructing the completion, we should first take the quotient space with respect to the partition of $\mathbf{N}$ into classes of points with zero distance. For example, the zero matrix leads to the one-point metric space. Thus finite metric spaces are also included in our considerations.

Now assume that we are given a Polish space $(X, \rho)$ equipped with a countable everywhere dense well-ordered subset $\left\{x_{i}\right\}_{i=1}^{\infty}$. The proper distance matrix $r=\left\{r_{i, j}\right\} \in$ $\mathcal{R}$, where $r_{i, j}=\rho\left(x_{i}, x_{j}\right), i, j=1,2, \ldots$, determines a metric on the set of positive 
integers. Thus the correspondence between proper distance matrices and equipped Polish spaces is a bijection. Every invariant property of the metric space (topological, homological, etc.) can be expressed in terms of the distance matrix of any countable everywhere dense set.

We may regard the cone $\mathcal{R}$ as a fiber bundle whose base space is the class of all individual Polish spaces (note that, by the universality of the Urysohn space (see below), we may assume that the base space is the set of all closed subsets of the Urysohn space) and the fiber over a given space is the set of all countable well-ordered everywhere dense subsets of this space. Thus the cone $\mathcal{R}$ is the universum of equipped Polish spaces, and we will study the properties of these spaces and the whole collection of them with the help of this cone.

Similarly to the definition of the cone $\mathcal{R}$, we can define the finite-dimensional cones $\mathcal{R}_{n}$ of distance matrices of order $n$. The cone $\mathcal{R}_{n}$ is a polyhedral cone lying inside the positive octant of the space of matrices $\operatorname{Mat}_{n}(\mathbf{R}) \equiv \mathbf{R}^{n^{2}}$. Let us define a subset $\mathbf{M}_{n}^{s}(\mathbf{R}) \equiv \mathbf{M}_{n}^{s}$ of the space of symmetric matrices as the set of symmetric matrices with zeros on the main diagonal. The cone $\mathcal{R}_{n}$ is contained in this set of matrices: $\mathcal{R}_{n} \subset \mathbf{M}_{n}^{s}$, and $\mathbf{M}_{n}^{s}$ is obviously the linear span of the cone: $\operatorname{span}\left(\mathcal{R}_{n}\right)=\mathbf{M}_{n}^{s}$, since the interior of $\mathcal{R}_{n}$ is not empty. Clearly, $\operatorname{span}(\mathcal{R}) \subset \mathbf{M}_{\mathbf{N}}^{s}$, where $\mathbf{M}_{\mathbf{N}}^{s}$ is the space of all real symmetric matrices with zeros on the main diagonal; the geometry of the cone $\mathcal{R}$ is quite nontrivial. Every distance matrix $r \in \mathcal{R}_{n}$ determines a (semi)metric on the space $X_{r}$ consisting of $n$ points.

Consider the projections

$$
p_{m, n}: \mathbf{M}_{m}^{s} \longrightarrow \mathbf{M}_{n}^{s}, \quad m>n,
$$

that associate with a matrix $r$ of order $m$ its NW-corner (= north-west corner) of order $n$. The cones $\mathcal{R}_{n}$ agree with these projections, i.e., $p_{m, n}\left(\mathcal{R}_{m}\right)=\mathcal{R}_{n}$. The projections $p_{n, m}$ extend naturally to a projection $p_{n}: M_{\mathbf{N}}^{s} \longrightarrow M_{n}^{s}(\mathbf{R})$ on the space of infinite symmetric matrices with zero diagonal, and the resulting projection $p_{n}$ also preserves the cones: $p_{n}(\mathcal{R})=\mathcal{R}_{n}$. It is clear that the cone $\mathcal{R}$, regarded as a topological space (in the weak topology), is the inverse limit of the sequence $\left(\mathcal{R}_{n},\left\{p_{n}\right\}\right)$.

An obvious but important property of the cones $\mathcal{R}_{n}$ is their invariance under the action of the symmetric group $S_{n}$ whose elements permute simultaneously the rows and columns of matrices.

Let us consider the geometric structure of the cones $\mathcal{R}_{n}$ and $\mathcal{R}$. In the first dimensions we have $\mathcal{R}_{1}=\{0\}$ and $\mathcal{R}_{2}=\mathbf{R}$. The description of extremal rays (in the sense of convex geometry) of the convex polyhedral cone $\mathcal{R}_{n}, n=3,4, \ldots, \infty$, is a well-known difficult combinatorial-geometric problem (see [12, 20, and references given there). Every extremal cone in $\mathcal{R}_{n}, n \leq 4$, is of the form $\{\lambda \cdot l: \lambda \geq 0\}$, where $l$ is a symmetric matrix with entries 0 and 1, i.e., the distance matrix associated with a semimetric such that the corresponding quotient space consists of two distinct points. But if $n \geq 5$, there are extremal rays consisting of proper metrics. A complete description of the set of extremal rays for the general dimension is not known. The most interesting question is the study of the asymptotic properties of the cones $\mathcal{R}_{n}$, and especially the description of the extremal rays of the cone $\mathcal{R}$. It may happen that the set of these rays is a dense $G_{\delta}$-set in $\mathcal{R}$ and some of them consist of universal distance matrices. This agrees 
with the estimates of the growth of the number of extremal rays of the cone $\mathcal{R}_{n}$ given in [20. It seems that the algebro-geometric structure and stratification of the cones $\mathcal{R}_{n}$ regarded as semialgebraic sets was not studied. In order to study the topological and convex structure of the cones $\mathcal{R}_{n}$, we will use their inductive description, which is given below.

\subsection{Admissible vectors and the structure of the cone $\mathcal{R}_{n}$}

Let $r=\left\{r_{i, j}\right\}_{1}^{n}$ be a distance matrix of order $n\left(r \in \mathcal{R}_{n}\right)$; choose a vector $a \equiv\left\{a_{i}\right\}_{i=1}^{n} \in$ $\mathbf{R}^{n}$ with the following property: if we attach it to the matrix $r$ as the last row and column, putting zero at the diagonal, we again obtain a distance matrix of order $n+1$. We say that such vectors are admissible for the given fixed distance matrix $r$ and denote the set of admissible vectors for $r$ by $A(r)$. Let $\left(r^{a}\right)$ stand for the distance matrix of order $n+1$ obtained from the matrix $r$ by attaching the vector $a \in A(r)$ as the last row and column; obviously, $p_{n}\left(r^{a}\right)=r$. The matrix $r^{a}$ is of the form

$$
r^{a}=\left(\begin{array}{ccccc}
0 & r_{1,2} & \ldots & r_{1, n} & a_{1} \\
r_{1,2} & 0 & \ldots & r_{2, n} & a_{2} \\
\vdots & \vdots & \ddots & \vdots & \vdots \\
r_{1, n} & r_{2, n} & \ldots & 0 & a_{n} \\
a_{1} & a_{2} & \ldots & a_{n} & 0
\end{array}\right)
$$

The (semi)metric space $X_{r^{a}}$ corresponding to the matrix $r^{a}$ is an extension of the space $X_{r}$ : we add one new point $x_{n+1}$, and the numbers $a_{i}, i=1, \ldots, n$, are the distances from $x_{n+1}$ to the old points $x_{i}$. The admissibility of the vector $a$ is equivalent to the following system of inequalities: an admissible vector $a=\left\{a_{i}\right\}_{i=1}^{n}$ for a fixed matrix $\left\{r_{i, j}\right\}_{i, j=1}^{n}$ must satisfy the series of triangle inequalities for all $i, j, k=$ $1,2, \ldots, n$ :

$$
\left|a_{i}-a_{j}\right| \leq r_{i, j} \leq a_{i}+a_{j}
$$

Thus for a distance matrix $r$ of order $n$, the set of admissible vectors is $A(r)=$ $\left\{\left\{a_{i}\right\}_{i=1}^{n}:\left|a_{i}-a_{j}\right| \leq r_{i, j} \leq a_{i}+a_{j}, i, j=1, \ldots, n\right\}$. It is worth mentioning that a vector $a=\left\{a_{i}\right\}$ may be regarded as a Lipschitz function $f_{a}(),. f_{a}(i)=a_{i}$, on the space $X_{r}=\{1,2, \ldots, n\}$ with metric $r$, with Lipschitz constant 1 . From this point of view it is useful to consider extensions of metric spaces (see [21, 4]).

Geometrically, the set of admissible vectors $A(r)$ can be identified with the intersection of the cone $\mathcal{R}_{n+1}$ and the affine subspace that consists of matrices of order $n+1$ whose NW-corner (north-west corner) coincides with the matrix $r$. Since the inequalities are linear, it is clear that the set $A(r)$ is an unbounded polyhedral convex set in $\mathbf{R}^{n}$. If $r_{i, j} \equiv 0, i, j=1, \ldots, n, n \geq 1$, then $A(r)$ is the diagonal: $A(0)=\Delta_{n} \equiv\{(\lambda, \ldots, \lambda): \lambda \geq 0\} \subset \mathbf{R}_{+}^{n}$. Let us describe the structure of the sets $A(r)$ in more detail.

Lemma 1 For every proper distance matrix $r$ of order $n$, the set of admissible vectors $A(r)$ is a closed polyhedral set lying in the octant $\mathbf{R}_{+}^{n}$, namely the Minkowski sum

$$
A(r)=M_{r}+\Delta_{n}
$$


where $\Delta_{n}$ is the half-line of constant vectors in the space $\mathbf{R}_{+}^{n}$ and $M_{r}$ is a convex compact polytope of dimension $n$, which is the convex hull of the set of extreme points of the whole polyhedron $A(r): M_{r}=\operatorname{conv}(\operatorname{ext} A(r))$.

Proof The set $A(r) \subset \mathbf{R}^{n}$ is the intersection of finitely many closed half-spaces and obviously does not contain lines. By general theorems of convex geometry, the set $A(r)$ is the sum of a convex polyhedron (polytope), which is the convex hull of the set of extreme points of the set $A(r)$, and a closed polyhedral cone, with vertex at the origin, that does not contain lines. It remains to observe that this cone degenerates into the diagonal of the positive octant, i.e., into the half-line of vectors in $\mathbf{R}^{n}$ with equal coordinates; indeed, if the cone contained another half-line, the triangle inequality would break: the difference $a_{i}-a_{j}$ of the coordinates could be arbitrarily large for at least one pair $(i, j)$. The dimension of the set $A(r)$ equals $n$ for a proper distance matrix; in the general case it depends on the matrix $r$ and can be less than $n$; the dimension of the polytope $M_{r}$ equals $\operatorname{dim} A(r)$ or $\operatorname{dim} A(r)-1$.

The following lemma asserts that the correspondence $r \rightarrow A(r)$ is covariant under the action of the symmetric group in $\mathbf{R}^{n}$. Its proof is obvious.

Lemma 2 For every matrix $r \in \mathcal{R}_{n}$, we have $A\left(\mathrm{grg}^{-1}\right)=g(A(r))$, where $g \in S_{n}$ is an element of the symmetric group $S_{n}$, which acts naturally on the space of matrices $M_{\mathbf{N}}(\mathbf{R})$ and on the space of convex subsets of the vector space $\mathbf{R}^{n}$.

The convex structure of the polyhedral sets $M_{r}$ and $A(r)$ is very interesting; apparently it has not been studied earlier. For dimensions greater than three, the combinatorial type of the polytope $M_{r}$ essentially depends on $r$. But in the three-dimensional case, this combinatorial type is the same for all proper distance matrices, and hence the type of the set $A(r)$ is also the same for all proper distance matrices. Let us consider this answer in more detail.

Example. For $n=3$, the set $A(r)$ and the extreme points of the polyhedron $M_{r}$ can be described as follows. Let $r$ be a proper distance matrix

$$
r=\left(\begin{array}{ccc}
0 & r_{1,2} & r_{1,3} \\
r_{1,2} & 0 & r_{2,3} \\
r_{1,3} & r_{2,3} & 0
\end{array}\right) .
$$

Set $r_{1,2}=\alpha, r_{1,3}=\beta, r_{2,3}=\gamma$; then

$$
r^{a}=\left(\begin{array}{cccc}
0 & \alpha & \beta & a_{1} \\
\alpha & 0 & \gamma & a_{2} \\
\beta & \gamma & 0 & a_{3} \\
a_{1} & a_{2} & a_{3} & 0
\end{array}\right)
$$

Let $\delta=\frac{1}{2}(\alpha+\beta+\gamma)$. There are seven extreme points $a=\left(a_{1}, a_{2}, a_{3}\right)$ of the polytope $A(r)$ : the first vertex is the closest vertex to the origin: $(\delta-\gamma, \delta-\beta, \delta-\alpha)$, three other ones are nondegenerate extreme points (metrics): $(\delta, \delta-\alpha, \delta-\gamma),(\delta-\beta, \delta, \delta-\alpha)$, $(\delta-\gamma, \delta-\beta, \delta)$, the remaining three vertices are degenerate: $(0, \alpha, \beta),(\alpha, 0, \gamma),(\beta, \gamma, 0)$. 
If $\alpha=\beta=\gamma=1$, then these seven points are as follows:

$(1 / 2,1 / 2,1 / 2),(3 / 2,1 / 2,1 / 2),(1 / 2,3 / 2,1 / 2),(1 / 2,1 / 2,3 / 2),(0,1,1),(1,0,1),(1,1,0)$.

Note that all nondegenerate extreme points determine metrics on the space consisting of four points that cannot be isometrically embedded into an Euclidean space.

\subsection{Projections and isomorphisms}

Let $r$ be a distance matrix of order $N$, and let $p_{n}(r)$ be its NW- (north-west) corner of order $n<N$. Then we can define a projection $\chi_{n}^{r}$ of the set $A(r)$ onto $A\left(p_{n}(r)\right)$ : $\chi_{n}^{r}:\left(b_{1}, \ldots, b_{n}, b_{n+1}, \ldots, b_{N}\right) \mapsto\left(b_{1}, \ldots, b_{n}\right)$. (We omit the index $N$ in the notation $\chi_{n}^{r}$.) The following simple lemma plays a very important role in our further construction.

Lemma 3 (Amalgamation lemma). Let $r \in \mathcal{R}_{n}$ be a distance matrix of order $n$. For any two vectors $a=\left(a_{1}, \ldots, a_{n}\right) \in A(r)$ and $b=\left(b_{1}, \ldots, b_{n}\right) \in A(r)$, there exists a real nonnegative number $h \in \mathbf{R}$ such that $\bar{b}=\left(b_{1}, \ldots, b_{n}, h\right) \in A\left(r^{a}\right)$ (and also $\left.\bar{a}=\left(a_{1}, \ldots, a_{n}, h\right) \in A\left(r^{b}\right)\right)$.

Corollary 1 For every matrix $r \in \mathcal{R}_{n}$ and every vector $a \in A(r)$, the mapping $\chi_{n+1, n}^{r}$ : $\left(b_{1}, \ldots, b_{n}, b_{n+1}\right) \mapsto\left(b_{1}, \ldots, b_{n}\right)$ of the set $A\left(r^{a}\right)$ into $A(r)$ is an epimorphism of the set $A\left(r^{a}\right)$ onto $A(r)$ (by definition, $p_{n+1, n}\left(r^{a}\right)=r$ ).

Proof The lemma follows from a simple geometric observation. Consider two finite metric spaces: $X=\left\{x_{1}, \ldots, x_{n-1}, x_{n}\right\}$ with metric $\rho_{1}$ and $Y=\left\{y_{1}, \ldots, y_{n-1}, y_{n}\right\}$ with metric $\rho_{2}$. Assume that the subspaces consisting of the first $n-1$ points $\left\{x_{1}, \ldots, x_{n-1}\right\}$ and $\left\{y_{1}, \ldots, y_{n-1}\right\}$ are isometric, that is, $\rho_{1}\left(x_{i}, x_{j}\right)=\rho_{2}\left(y_{i}, y_{j}\right), i, j=1, \ldots, n-1$. Then there exists a third space $Z=\left\{z_{1}, \ldots, z_{n-1}, z_{n}, z_{n+1}\right\}$ with metric $\rho$ and isometries $I_{1}$ and $I_{2}$ of the spaces $X$ and $Y$, respectively, into the space $Z$ such that

$$
I_{1}\left(x_{i}\right)=z_{i}, I_{2}\left(y_{i}\right)=z_{i}, i=1, \ldots, n-1, I_{1}\left(x_{n}\right)=z_{n}, I_{2}\left(y_{n}\right)=z_{n+1} .
$$

In order to prove the existence of such a space $Z$, we must show that there exists a nonnegative number $h$ that can be the distance $\rho\left(z_{n}, z_{n+1}\right)=h$ between $z_{n}$ and $z_{n+1}$ (the images of $x_{n}$ and $y_{n}$, respectively, in $Z$ ), i.e., such a distance $h$ must satisfy all triangle inequalities in the space $Z$. The existence of such a number $h$ follows from the inequalities

$$
\begin{aligned}
& \rho_{1}\left(x_{i}, x_{n}\right)-\rho_{2}\left(y_{i}, y_{n}\right) \leq \rho_{1}\left(x_{i}, x_{j}\right)+\rho_{1}\left(x_{j}, x_{n}\right)-\rho_{2}\left(y_{i}, y_{n}\right) \\
= & \rho_{1}\left(x_{j}, x_{n}\right)+\rho_{2}\left(y_{i}, y_{j}\right)-\rho_{2}\left(y_{i}, y_{n}\right) \leq \rho_{1}\left(x_{j}, x_{n}\right)+\rho_{2}\left(y_{j}, y_{n}\right),
\end{aligned}
$$

which hold for all $i, j=1, \ldots, n-1$. Therefore,

$$
\max _{i}\left|\rho_{1}\left(x_{i}, x_{n}\right)-\rho_{2}\left(y_{i}, y_{n}\right)\right| \equiv M \leq m \equiv \min _{j}\left(\rho_{1}\left(x_{j}, x_{n}\right)+\rho_{2}\left(y_{j}, y_{n}\right)\right) .
$$

Thus we can take $h$ equal to an arbitrary number from the nonempty closed interval $[M, m]$, and we set $\rho\left(z_{n}, z_{n+1}\right) \equiv h$; it follows from the definition of $h$ that all triangle 
inequalities are satisfied. Now assume that $r$ is a distance matrix of order $n-1$ and $a \in$ $A(r)$ is an admissible vector; let $\left\{x_{1}, \ldots, x_{n-1}, x_{n}\right\}$ be a space such that the distances between the first $n-1$ points are given by the matrix $r$ and the metric on the whole space is given by the extended matrix $r^{a}$. We can choose another arbitrary admissible vector $b \in A(r)$ and obtain the distance matrix $r^{b}$ of a space $\left\{y_{1}, \ldots, y_{n-1}, y_{n}\right\}$, where the space consisting of the first $n-1$ points is isometric to the space $\left\{x_{1}, \ldots, x_{n-1}\right\}$. As we have proved above, there exists a space $Z$ whose distance matrix $\bar{r}$ of order $n+1$ enjoys the desired property.

"Amalgamation lemmas," i.e., lemmas on extension of homomorphisms of two spaces $X_{1}$ and $X_{2}$ to a space $X_{3}$ with an identified subspace $X_{4}$ to a homomorphism of their union to $X_{3}$, are an element of the construction of the inductive limit that is known in the theory of models as the Fraisse limit. We apply the term "amalgamation" exactly in the same context in which it is used in the theory of models, when one studies the possibility of embedding two intersecting but noncoinciding models into a third one. In fact, this lemma predetermines the existence of the universal object in the category, in our case - the universal metric space. In the next section we will use the inequality we have proved in the inductive construction of measures.

Now we are able to state a general assertion on the projection $\chi^{r}$.

Lemma 4 For arbitrary positive integers $N$ and $n<N$ and every matrix $r \in \mathcal{R}_{N}$, the mapping $\chi_{n}^{r}$ is an epimorphism of the set $A(r)$ onto $A\left(p_{n}(r)\right)$. In other words, for every $a=\left(a_{1}, \ldots, a_{n}\right) \in A\left(p_{n}(r)\right)$, there exists a vector $\left(b_{n+1}, \ldots, b_{N}\right)$ such that $b=\left(a_{1}, \ldots, a_{n}, b_{n+1}, \ldots, b_{N}\right) \in A(r)$.

Proof The proof of the previous lemma shows how we should determine the first number $b_{n+1}$. But $\chi_{n}^{r}$ is a mapping of the set $A(r), r \in \mathcal{R}_{N}$, to the similar set $A\left(p_{n}(r)\right)$ and is the product of the projections $\chi_{n}^{r}, \cdots, \chi_{N-1}^{r}$, each of them being an epimorphism; hence the product is also epimorphic.

In what follows, it is convenient to represent an infinite matrix $r \equiv\left\{r_{i, j}\right\} \in \mathcal{R}$ as a sequence of admissible vectors of increasing lengths

$r(1)=\left\{r_{1,2}\right\}, r(2)=\left\{r_{1,3}, r_{2,3}\right\}, \ldots, r(k)=\left\{r_{1, k+1}, r_{2, k+1}, \ldots, r_{k, k+1}\right\}, \ldots, k=1,2, \ldots$,

satisfying the conditions $r(k) \in A\left(p_{k}(r)\right.$ ) (recall that $p_{k}(r)$ is the NW-projection of a matrix $r$ to the space $\mathbf{M}_{k}^{s}$, i.e., the NW-corner of $r$ ), that is, such that every vector $r(k)$ is admissible for the previous distance matrix $p_{k}(r)$.

We may consider the following sequence of cones and mappings:

$$
0=\mathcal{R}_{1} \stackrel{p_{2}}{\longleftarrow} \mathcal{R}_{2}=\mathbf{R}_{+} \stackrel{p_{3}}{\longleftarrow} \mathcal{R}_{3} \longleftarrow \ldots \longleftarrow \mathcal{R}_{n-1} \stackrel{p_{n}}{\longleftarrow} \mathcal{R}_{n} \longleftarrow \ldots ;
$$

here $p_{n}$ is the restriction of the projection defined above to the cone $\mathcal{R}_{n}$. The preimage of a point $r \in \mathcal{R}_{n-1}$ (the fiber over $r$ ) is the set $A(r)$ defined in Lemma 1. Note that the sequence (3) is not a sequence of fiber bundles in the ordinary sense: the preimages may be even nonhomeomorphic for different points $r$ (even their dimensions may be different). But this sequence determines the cone $\mathcal{R}$ as the inverse limit of the cones $\mathcal{R}_{n}$. We use the sequence (3) to define measures on the cone $\mathcal{R}$ in the spirit of the theory of Markov processes. 


\subsection{Definition of the "most" random metric space}

Using the geometry of the cone of distance matrices, we will define a class of Borel probability measures on this cone that may be regarded as the most "dispersed" distributions, as far as the cone of distance matrices allows. A random distance matrix governed by this distribution corresponds to the intuitive notion of a random metric space in the following sense, which will be specified below: the set of supports of the distributions of finite fragments of this random matrix coincides with the set of all distance matrices of finite metric spaces without any restrictions. Since the cone $\mathcal{R}$ is infinite-dimensional, it has no distinguished measure (similar to the Lebesgue measure on a finite-dimensional manifold); hence we should speak of the class of most dispersed measures, rather than of a single measure. This class, as we will see, includes measures with very different behavior. On the other hand, in view of the triangle inequality, we cannot (except for the example described below) assume that all pairwise distances are independent. We construct measures with the help of a Markovian procedure, which imitates independence and is useful in other cases. Briefly speaking, we construct a random matrix as a random two-dimensional field, Markovian in some sense.

The construction is inductive: we begin with the one-point space and then describe how to add a new $(n+1)$ th point to $n$ points already constructed, choosing the distances from the new point to the old ones in the most random way. The "maximum randomness" means that, given a distance matrix $r_{n} \in \mathcal{R}_{n}$ already chosen, we choose an admissible vector uniformly distributed on $A\left(r_{n}\right)$. This method corresponds to the literal understanding of what is a random finite metric space.

After infinitely many steps we obtain an infinite random distance matrix, i.e., a probability measure on the cone $\mathcal{R}$, or, in other words, a random metric on the set of positive integers. Then the following question arises: what metric spaces are the completions of the set of positive integers with respect to these metrics? We will answer this question in Section 3. Now we mention only that this is one and the same, up to isometry, metric space for almost all (in the sense of the measure constructed) distance matrices, and almost all matrices are the distance matrices of various everywhere dense countable sequences of points in this space; this space is precisely the universal Urysohn space.

Let us now describe the precise construction of probability measures on the cone $\mathcal{R}$. The parameter of this construction is an arbitrary continuous probability measure $\gamma$ of full support on the half-line $\mathbf{R}_{+}^{1}$, for example the Gaussian measure on the half-line. Consider the one-point metric space, and assume that its single point is the positive integer 1 . Every time when we add a new point $n, n=2,3, \ldots$, we let its distance $r_{1, n}$ to the point 1 be equal to the random variable $\xi_{n}, n=2,3, \ldots$, and assume that the random variables $\xi_{n}$ for different $n$ are i.i.d. with distribution $\gamma$. Thus the distribution of the first row (and the first column) of the random matrix is defined; in particular, the distribution of the entry $r_{1,2}$ is defined.

Now we define a measure $\nu_{\gamma}$ on the cone $\mathcal{R}$ by induction. We use the following method of describing a measure: fix $n>2$ and $k<n$, and assume that we are given a probability distribution on the fragments of finite rectangular distance matrices, namely on the set of collections $\left\{r_{i, j}\right\}$, where the indices range over the sets $i, j=1, \ldots, n-1$ and $(i, n), i=1, \ldots, k$; in other words, we are given the joint distribution of the 
pairwise distances between all pairs of the first $n-1$ points and the distances from the $n$th point to the first $k$ points, $1<k<n$. Now let us fix the values of these distances and determine the conditional distribution of the distance between the points $n$ and $k+1$.

Obviously, the unique inequality satisfied by the distance $r_{k+1, n}$ is

$$
\max _{i=1, \ldots, k}\left|r_{i, k+1}-r_{i, n}\right| \leq r_{k+1, n} \leq \min _{i=1, \ldots, k}\left|r_{i, k+1}+r_{i, n}\right| \text {. }
$$

The amalgamation lemma from the previous section implies that the left-hand side of the inequality does not exceed the right-hand side, so that the range of possible values for $r_{k+1, n}$ is not empty. As the conditional measure for $r_{k+1, n}$, take the Lebesgue measure on this interval normalized to one. Of course, the interval depends substantially on the matrix fragment we consider; hence our measure is not (and, as mentioned above, cannot be for any change of parameters) a product measure: the pairwise distances are not independent. However, one should regard the above definition of conditional measures as the best admissible step-wise replacement of the independence of $r_{k+1, n}$ and the previous distances. When $k$ assumes the value $n-1$ (recall that $r_{n, n}=0$ ), the distribution of the matrix $r_{i, j}, i, j=1, \ldots, n, n>2$, is defined, i.e., we obtain a random $n$-point metric space. Since the distribution of the entry $r_{1, n+1}$ is already determined, we define, according to the same rules, the conditional measure of the entry $r_{2, n+1}$, i.e., the distance between the points 2 and $n+1$. The description of the algorithm is finished.

Our method of constructing a measure, which can be called Markovian, is based on the following general fact from measure theory: if we are given a measurable partition (above it was the one-dimensional fiber bundle over the described fragment of the matrix with fiber $r_{k+1, n}$ ) in a standard measure space (Lebesgue-Rokhlin space), then a measure on the whole space is uniquely determined by its projection to the base space (i.e., a measure on the quotient space) and the system of conditional measures on fibers (for almost all fibers). The fiber of our fiber bundle is the interval of possible values for the distance between the $(k+1)$ th and $n$th points, given fixed (already determined) distances between the pairs of points with numbers less than $n$ and between the $n$th and $i$ th points for $i<k+1$.

Thus the distribution of the entries of the first row and the above construction of conditional measures uniquely determine the measure $\nu_{\gamma}$.

Lemma 5 (on stationarity).

1. The support of the measure $\nu_{\gamma}$ is the whole cone $\mathcal{R}$.

2. Denote by $\nu_{\gamma}^{n}$ the projection of the measure $\nu_{\gamma}$ to the set of submatrices $\left\{\left\{a_{i, j}\right\}\right.$ : $i=1, \ldots, n ; j=n+1, n+2, \ldots\}$, i.e., the joint distribution of these matrix entries. The measure $\nu_{\gamma}^{n}$ is invariant under the one-sided shift $\left\{a_{i, j}\right\} \rightarrow\left\{a_{i, j+1}\right\}$ and ergodic.

Proof Both claims follow immediately by induction from the construction of the measure. The projection of the measure $\nu_{\gamma}$ to the first row (column) is of full support, i.e., its support coincides with $\left(\mathbf{R}_{+}\right)^{\infty}$. In order to check this, it suffices to recall that the sequence of entries of the first row is a sequence of i.i.d. variables on $\mathbf{R}_{+}$with distribution $\gamma$, and hence this sequence is stationary. Assume that we have proved 
that the projection is of full support and the sequence is stationary for the first $k$ rows. Since in the above inequality determining the distribution of the entry $r_{k+1, n}$, both left- and right-hand sides depend only on the entries of the previous $k$ rows and this dependence is invariant under the horizontal shift in $n$ for fixed $k$, it follows that the left- and right-hand sides of the inequality determine stationary sequences of random variables. The choice of the entry $r_{k+1, n}$ reduces to independently choosing a number uniformly distributed in the interval between the boundaries of the inequality. Thus the elements of the sequence $\left\{r_{k+1, n}\right\}_{n=k+1}^{\infty}$ ( $k$ is fixed) are functions (which do not depend on $n$ ) of several stationary sequences, namely the previous rows and a sequence of independent variables; hence the first $k+1$ rows form a stationary random sequence in $n, n=k+1, k+2, \ldots$. Obviously, the shift of the first row is ergodic; the induction step proceeds as follows: it is easy to see that the shift acting in the space of $(k+1)$-rows is the factor shift of the direct product of the shift acting in the space of $k$-rows and a Bernoulli shift (that determines the choice of the entries $r_{k+1, n}, n=k+1, k+2, \ldots$, in the intervals; see above); hence it is ergodic. The same considerations imply that the projection of the measure is of full support.

As we have already mentioned, this method of defining measures on the set of matrices can be called Markovian, because we can also regard the conditional measures defined above as the transition probabilities of a random field if we will regard the random matrix itself as a random field on the lattice $\left(\mathcal{Z}_{+}\right)^{2}$ with boundary condition given by the distribution of the first row and the first column. The procedure described above is simpler than that given in [6], though they are essentially equivalent. The measure $\gamma$ on the half-line, as well as the conditional measures, may vary in wide ranges; however, these variations are inessential; only explicit conditions on the random distance matrix are of importance; they correspond to the intuitive notion of randomness and guarantee the conditions of the theorem from Section 3, which we will check with the help of the lemma proved above.

An illustrative description of our construction of the measure is as follows: given an $n$-point metric space, we add the next $(n+1)$ th point at random, the vector of distances between the new point and the old ones (i.e., an admissible vector) having a uniformly positive probability on the set of admissible vectors. Thus this procedure can be used for simulating a generic metric space, as in the Monte-Carlo method. We must warn those who dealt with experiments with random configurations that metric spaces having an isometric realization in an Euclidean space form an exponentially small fraction with respect to our measure as $n \rightarrow \infty$.

Let us supplement the construction with several remarks.

1. If we impose an additional condition on all distances: $r_{i, j} \leq b$, then the same procedure (in this case we may assume, for example, that $\gamma$ is the uniform measure on $[0, b])$ will give us a random metric space of diameter $b$.

2. An obvious drawback of our construction is that it is not invariant with respect to the numbering of points; for example, the first point is distinguished: the sequence of distances from the first point to the other ones is a sequence of independent variables, but the sequence of distances from the second (or any subsequent) point to the other ones is no longer a sequence of independent variables. The situation can be remedied 
by symmetrization, i.e., by considering the weak limit of measures

$$
\bar{\nu}_{\gamma}=\lim (n !)^{-1} \sum_{g \in S_{n}} g \nu_{\gamma},
$$

where the symmetric group acts by simultaneous permutations of the rows and columns of a distance matrix (and hence on measures defined on the cone of distance matrices). It is not difficult to prove that the weak limit $\bar{\nu}_{\gamma}$ exists and, obviously, is invariant under the action of the infinite symmetric group. However, this gives rise to a new series of questions, which are partially discussed in Section 5.

3. One can use the same recipe to construct arbitrary measures on matrices: first one should determine the distribution of the first row (column) and then successively determine the conditional measures of entries. This method applies to constructing not only random distance matrices, but also measures on other families of matrices.

\section{Universal distance matrices and the Urysohn space. Urysohnness criterion}

\subsection{Definitions and the urysohnness criterion}

The following definition plays a key role in our considerations.

Definition 1 1. An infinite distance matrix $r=\left\{r_{i, j}\right\}_{i, j=1}^{\infty} \in \mathcal{R}$ is called a universal distance matrix if the following condition holds:

for every $\epsilon>0$, every $n \in \mathbf{N}$, and every vector $a=\left\{a_{i}\right\}_{i=1}^{n} \in A\left(p_{n}(r)\right)$, there exists $m \in \mathbf{N}$ such that $\max _{i=1, \ldots, n}\left|r_{i, m}-a_{i}\right|<\epsilon$.

In other words, for every $n \in \mathbf{N}$, the set of vectors $\left\{\left\{r_{i, j}\right\}_{i=1}^{n}\right\}_{j=n+1}^{\infty}$ is everywhere dense in the set of admissible vectors $A\left(p_{n}(r)\right)$.

2. An infinite proper distance matrix $r=\left\{r_{i, j}\right\}_{i, j=1}^{\infty} \in \mathcal{R}$ is called an almost universal distance matrix if for every $n \in \mathbf{N}$, the set of all its submatrices of order $n$ of the form $\left\{r_{i_{k}, i_{s}}\right\}_{k, s=1}^{n}$ over all $n$-tuples $\left\{i_{k}\right\}_{k=1}^{n} \subset \mathbf{N}$ is dense in the cone $\mathcal{R}_{n}$.

Denote the set of universal distance matrices (respectively, almost universal distance matrices) by $\mathcal{M}$ (respectively, $\mathcal{M}^{\prime}$ ). We will prove that $\mathcal{M}$ is not empty, but first we describe some properties of universal matrices.

Lemma 6 Every universal distance matrix is almost universal. There exists an almost universal nonuniversal distance matrix.

Proof Let $r$ be a universal matrix; let us choose an arbitrary finite distance matrix $q \in \mathcal{R}_{n}$ and prove that, given a positive number $\epsilon$, we can find a set $\left\{i_{k}\right\}_{k=1}^{n} \subset \mathbf{N}$ such that $\max _{k, s=1, \ldots, n}\left|r_{i_{k}, i_{s}}-q_{k, s}\right|<\epsilon$. Since $r^{1}=\left\{r_{1,1}=0\right\}$, we have $A\left(r^{1}\right)=\mathbf{R}_{+}$(see Section 2.2); since $r$ is universal, the sequence $\left\{r_{1, n}\right\}_{n=2}^{\infty}$ is dense in $\mathbf{R}_{+}$; therefore we can choose a positive integer $i_{1}$ such that $\left|r_{1, i_{1}}-q_{1,2}\right|<\epsilon$ and then, using the density of columns of length two, which follows from the universality condition, choose a positive 
integer $i_{2}$ such that $\left|r_{1, i_{2}}-q_{1,3}\right|<\epsilon,\left|r_{2, i_{2}}-q_{2,3}\right|<\epsilon$; iterating this procedure for successive columns of the matrix $q \in \mathcal{R}_{n}$, we obtain a desired submatrix of $r$.

There are many examples of almost universal matrices that are not universal; in particular, the distance matrix of every countable everywhere dense subset of the universal, but not homogeneous (see below) Polish space (for example, the Banach space $C([0,1]))$ yields such an example.

Corollary 2 (on $\epsilon$-extension of isometries). Let $r$ be an infinite distance matrix. The matrix $r$ is universal if and only if for every positive $\epsilon$, every positive integer $n<N$, and every finite distance matrix $q \in \mathcal{R}_{N}$ of order $N$ whose $N W$-corner of order $n$ coincides with the $N W$-corner of the same order of the matrix $r$ (i.e., $r_{i, j}=q_{i, j}$, $i, j=1, \ldots, n)$, there exist positive integers $i_{1}, \ldots, i_{N}$ with $i_{k}=k, k=1, \ldots, n$, such that $\max _{k, s=1, \ldots, N}\left|r_{i_{k}, i_{s}}-q_{k, s}\right|<\epsilon$.

In other words, we can extend the set of the first $n$ positive integers with an $(N-n)$ tuple of positive integers $i_{n+1}, \ldots, i_{N}$ in such a way that the resulting distance matrix of order $N$ with indices $i_{1}=1, i_{2}=2, \ldots, i_{n}=n, i_{n+1}, \ldots, i_{N}$ will differ from the distance matrix $q$ (with respect to an arbitrary given norm) by less than $\epsilon$.

The property stated in the first claim of the corollary is called the $\epsilon$-extension of isometries, because it means that an isometry between the first $n$ points of a finite metric space of cardinality $N$ and a countable metric space with a given distance matrix can be extended to an $\epsilon$-isometry of the whole finite metric space.

Proof Assume that $r$ is a universal matrix and $N=n+1$; then the assertion follows immediately from the definition of universality; the desired assertion follows by induction on $N$. And if the condition of the corollary holds for $N=n+1$, it follows that the matrix is universal.

Another useful reformulation of the notion of universality and almost universality, which follows from the corollary, involves group-theoretic terms. Let $q \in \mathcal{R}_{n}$; denote by $\mathcal{R}^{n}(q)$ the set of all matrices $r \in \mathcal{R}$ such that the NW-corner of $r$ coincides with $q$. Consider the group $S_{\infty}^{n}$ of simultaneous permutations of rows and columns of matrices that leave the first $n$ rows and columns of distance matrices invariant and hence send $\mathcal{R}^{n}(q)$ to itself. The following universality criterion follows immediately from definitions.

Proposition 1 A matrix $r \in \mathcal{R}$ is universal if and only if for every positive integer $n$, its orbit under the action of the group $S_{\infty}^{n}$ is everywhere dense in $\mathcal{R}^{n}\left(r^{n}\right)$ in the weak topology (here $r^{n}$ is the $N W$-corner of order $n$ of the matrix $r$ ). A matrix $r \in \mathcal{R}$ is almost universal if and only if for every $n$, its orbit under the action of the whole group $S_{\infty}$ is everywhere dense in the cone $\mathcal{R}$.

The existence of universal distance matrices is not quite obvious; as we will see, it is equivalent to the existence of the universal Urysohn space. Urysohn's work [1, 2, 3] begins with a direct construction of a concrete universal rational distance matrix, though he uses neither this notion, nor its properties. We slightly simplify Urysohn's proof from 2] and prove not only the existence of universal matrices, but also a more general fact: 
Theorem 1 The set $\mathcal{M}$ of universal matrices is not empty and is an everywhere dense $G_{\delta}$-set in the cone $\mathcal{R}$ (in the weak topology).

Before proving the theorem, note that the random distance matrix constructed in the previous section is universal with probability one; this fact will be proved in the next section; thus, in particular, the existence of universal matrices will be proved. In this section we will give a deterministic version of the same inductive construction of a universal matrix.

Proof Let us first prove the existence of universal distance matrices in the cone $\mathcal{R}$; we use the inductive method and the notions defined in the previous sections.

As the first row of the matrix, take an arbitrary sequence $\left\{r_{1, n}\right\}_{n=1}^{\infty}$ everywhere dense in $\mathbb{R}_{+}$. Since $r_{1,1}=0$ and $A(0)$ coincides with $\mathbb{R}_{+}$, the universality condition for the first row is satisfied. Assume that we have already constructed the first $k-1$ rows satisfying the desired conditions. Let us define the $k$ th row $\left\{r_{k, n}\right\}_{n=k+1}^{\infty}$ elementwise, beginning (since the matrix is symmetric) with the entry $r_{k, k+1}$. By the induction hypothesis, the column vectors $\left\{r_{i, j}\right\}_{i=1}^{k-1}$ for all $j=k+1, k+2, \ldots$ lie in the admissible set $A\left(r^{k-1}\right)$, where $r^{k-1}$ is the submatrix of order $k-1$ (the NW-corner of order $k-1$ ) of the matrix $r$, and the sequence of these columns is dense in the admissible set $A\left(r^{k-1}\right)$. We must supplement the columns with numbers $\left\{r_{k, j}\right\}_{j=k+1}^{\infty}$ so that every resulting column will lie in the set $A\left(r^{k}\right)$ and the whole set of them will be dense in $A\left(r^{k}\right)$. The existence of such numbers is an immediate consequence of the following elementary lemma on the "lifting" of a countable everywhere dense set from the base space of a fiber bundle to the bundle itself.

Lemma 7 Let $K$ be a closed convex set, in the Euclidean space $\mathbb{R}^{k}$, represented as a fiber bundle over its projection $K_{0}$ to the coordinate hyperplane $\mathbb{R}^{k-1}$, the fiber over a point of $K_{0}$ (the preimage of a point under the projection) being a closed interval of the real axis. For every countable everywhere dense set $\left\{y_{n}\right\}_{n \in \mathrm{N}}$ in $K_{0}$, there exists a sequence of real numbers $\left\{z_{n}\right\}_{n \in \mathrm{N}}$ such that every vector $\left(y_{n}, z_{n}\right)$ belongs to the cone $K$ (in other words, $z_{n}$ lies in the interval-fiber over the point $y_{n}$ ) and the set of all such vectors is everywhere dense in $K$.

Proof Indeed, since the fiber over the point $y_{n}$ is a nonempty interval $\left[a_{n}, b_{n}\right]$, we can choose a finite or countable everywhere dense set $\left\{z_{i, n}\right\}_{i}$ in each of these intervals. Obviously, we can define the points $z_{n}=z_{i(n), n}$ corresponding to the points $y_{n}$ by induction in such a way that the countable set $\left\{\left(y_{n}, z_{n}\right)\right\}_{i}$ will be everywhere dense in $K$. We omit standard details.

We apply this lemma as follows: let $K=A\left(r^{k}\right)$; then its projection $K_{0}$ is $A\left(r^{k-1}\right)$, and the fiber over a point $\left\{r_{i, j}\right\}_{i=1, \ldots, k-1} \in A\left(r^{k-1}\right)$ is the interval of possible values for the matrix entry $r_{k, j}, j=k+1, k+2, \ldots$. The existence of universal matrices is proved.

Since the universality of a distance matrix is a property that is preserved under simultaneous finite permutations of rows and columns, as well as under the NW-shift (see above), and moreover it is preserved under a change of finitely many matrix entries, it follows that the set $\mathcal{M}$ contains, together with each matrix, all its permutations and NW-shifts. Since universality implies almost universality, it follows that the $S_{\mathbf{N}}$-orbit 
of a universal matrix $r$ is everywhere dense in $\mathcal{R}$ in the weak topology. Thus $\mathcal{M}$ is everywhere dense in $\mathcal{R}$.

Finally, the following formula is an immediate consequence of the definition of universality and shows that the set $\mathcal{M}$ of universal matrices is an everywhere dense $G_{\delta}$-set:

$$
\mathcal{M}=\bigcap_{k \in \mathbf{N}} \bigcap_{n \in \mathbf{N}} \bigcap_{a \in A\left(r^{n}\right) \cap \mathbf{Q}^{n^{2}}} \bigcup_{m \in \mathbf{N}, m>n}\left\{r \in \mathcal{R}: \max _{i=1, \ldots, n}\left|r_{i, m}-a_{i}\right|<\frac{1}{k}\right\} .
$$

Fix a universal proper distance matrix $r$ and equip the set of all positive integers $\mathbf{N}$ with the metric $r$. Denote by $\left(\mathcal{U}_{r}, \rho_{r}\right)$ the completion of the metric space $(\mathbf{N}, r)$ with respect to the metric $r$. It is obviously a Polish space.

Lemma 8 The distance matrix of every countable everywhere dense subset $\left\{u_{i}\right\}$ of the space $\mathcal{U}_{r}$ is universal.

Proof Identify the set of positive integers $\mathbf{N}$ with the set $\left\{x_{i}\right\} \subset \mathcal{U}_{r}$. By definition, $\rho\left(x_{i}, x_{j}\right)=r_{i, j}$. Since $r$ is a universal matrix, it follows that for every $n$, the following property holds (where $\mathrm{Cl}$ stands for the closure): $\mathrm{Cl}\left(\cup_{j>n}\left\{\left\{\rho\left(x_{i}, x_{j}\right)\right\}_{i=1}^{n}\right\}=A\left(p_{n}(r)\right)\right.$. Since the set $\left\{u_{i}\right\}$ is everywhere dense in $\left(\mathcal{U}_{r}, \rho_{r}\right)$, we may replace the last inequality with the following one: $\operatorname{Cl}\left(\cup_{j>n}\left\{\left\{\rho\left(x_{i}, u_{j}\right)\right\}_{i=1}^{n}\right\}=A\left(p_{n}(r)\right)\right.$. But since $\left\{x_{i}\right\}$ is also everywhere dense in $\left(U_{r}, \rho_{r}\right)$, we can write $\mathrm{Cl}\left(\cup_{j>n}\left\{\left\{\rho\left(u_{i}, u_{j}\right)\right\}_{i=1}^{n}\right\}=A\left(p_{n}\left(r^{\prime}\right)\right)\right.$, where $r^{\prime}$ is the distance matrix of the sequence $\left\{u_{i}\right\}$.

Thus the universality of the distance matrix of a countable dense subset is a property of the Polish space - it does or does not hold for all such subsets simultaneously. We will see that the space $\left(\mathcal{U}_{r}, \rho_{r}\right)$ is the so-called universal Urysohn space (which is defined below) and the universality of a distance matrix is a necessary and sufficient condition that guarantees that the completion of the set of positive integers with respect to the corresponding metric is isometric to the Urysohn space.

\subsection{Universal Urysohn space and universal matrices}

Now let us define the Urysohn space. In his last paper [2, which was published already after his tragic death, Pavel Urysohn (1898-1924) suggested a construction of the universal Polish space, which is now called the "Urysohn space." This paper was an answer to the question, posed by M. Fréchet, on the existence of the universal Banach space. Later Banach and Mazur gave a direct answer to Fréchet's question by showing that there exists a universal Banach space (for example, $C([0,1])$ ); their result is a simple consequence of a theorem due to Hausdorff. In the book [1], Urysohn's paper is mentioned, but without indicating the most important properties of the Urysohn space: homogeneity and uniqueness up to isometry. But precisely these properties impart a fundamental character to this space. We unify and slightly generalize the main theorems of the paper [2] in the following statement. 
Theorem 2 (Urysohn [1, 2])

There exists a Polish (= complete separable metric) space $\mathcal{U}$ with the following properties:

1) (Universality.) For every Polish space $X$, there exists an isometric embedding of $X$ into $\mathcal{U}$.

2) (Homogeneity.) For any two isometric finite subsets $A=\left(a_{1}, \ldots, a_{m}\right)$ and $B=$ $\left(b_{1}, \ldots, b_{m}\right)$ of the space $\mathcal{U}$, there exists an isometry $J$ of the whole space $\mathcal{U}$ that sends A to $B: J A=B$.

3) (Uniqueness.) Any two Polish spaces that satisfy conditions 1) and 2) are isometric.

Under the conditions of the theorem, property 2) can be strengthened: finite subsets can be replaced with compact ones; thus the group of isometries of the space acts transitively on isometric compact subsets; however, compact subsets in this statement cannot be replaced with closed ones.

Condition 2) can be strengthened in a similar way and formulated as a condition of extension of a given isometry of two compact subsets to the whole space; more precisely:

$\left.2^{\prime}\right)$ Let $F$ be a finite subset in $\mathcal{U}$, and let $i$ be an isometric embedding of $F$ into a finite metric space $F^{\prime}$. Then there exists an isometric embedding $I$ of the space $F^{\prime}$ into $\mathcal{U}$ such that the product $I \cdot i$ is the identity mapping of the subset $F$.

Below we will prove the homogeneity in this strengthened form. In this condition we can also replace finite sets $F$ and $F^{\prime}$ with compact ones.

Note that there are many distinct (nonisometric) universal, but not homogeneous Polish spaces (for example, the Banach space $C([0,1])$ ). The above corollary shows that the principal difference between such universal spaces and the Urysohn space is as follows: any Polish space can be isometrically embedded in any universal space; but in the case of the Urysohn space, one can do much more: given a fixed finite (and even compact) subset of the Urysohn space, the images of the points of the Polish space under the embedding can have any prescribed distances to the points of this subset. Similarly, the homogeneity property holds, for example, in Euclidean and Hilbert spaces. But the two properties simultaneously determine the space uniquely up to isometry.

The main result of this section is the following theorem, which includes the previous one.

Theorem 3 1. The completion $\left(\mathcal{U}_{r}, \rho_{r}\right)$ of the space of positive integers $(\mathbf{N}, r)$ with respect to the metric determined by a universal proper distance matrix $r$ satisfies properties 1), 2), and 2') of the previous theorem, i.e., is the Urysohn space.

2. (Uniqueness.) For any two universal proper distance matrices $r$ and $r^{\prime}$, the completions of the spaces $(\mathbf{N}, r)$ and $\left(\mathbf{N}, r^{\prime}\right)$ are isometric. Thus the isometric type of the space $\left(\mathcal{U}_{r}, \rho_{r}\right)$ does not depend on the choice of the universal matrix $r$. The universality is a necessary and sufficient condition on the distance matrix of any countable everywhere dense subset of the Urysohn space. 
The proof of Theorem 3 given below partially reproduces and simplifies the arguments of Urysohn's paper. Urysohn did not use infinite distance matrices. We will essentially follow our paper [6].

Proof Assume that $r=\{r(i, j)\}_{i, j=1}^{\infty} \in \mathcal{R}$ is a proper universal distance matrix (it is convenient to write $r(i, j)$ instead of $\left.r_{i, j}\right)$ and $\mathcal{U}_{r}$ is the completion of the countable metric space $(\mathbf{N}, r)$. We denote the corresponding metric on $\mathcal{U}_{r}$ by $\rho_{r}$, sometimes omitting the index $r$.

1. First of all, let us prove that the metric space $(\mathcal{U}, \rho)$ is universal in the sense of property 1) from Theorem 2 and that it is homogeneous in the sense of property 2 ) from the same theorem.

Let $(Y, q)$ be an arbitrary Polish space. In order to prove that there exists an isometric embedding of $(Y, q)$ into $(\mathcal{U}, \rho)$, it suffices to prove that there exists an isometric embedding of an arbitrary countable dense subset $\left\{y_{n}\right\}_{1}^{\infty}$ of the space $(Y, q)$ into $(\mathcal{U}, \rho)$. Thus we must prove that for every infinite proper distance matrix $q=\{q(i, j)\} \in \mathcal{R}$, there exists a dense subset $\left\{u_{i}\right\} \subset \mathcal{U}$ with distance matrix equal to $q$. This in turn means that we must construct a set of fundamental sequences in the space $(\mathbf{N}, r)$, say $N_{i}=\left\{n_{i}^{(m)}\right\}_{m=i}^{\infty} \subset \mathbf{N}, i=1,2, \ldots$, such that

$$
\begin{aligned}
\lim _{m \rightarrow \infty} r\left(n_{i}^{(m)}, n_{j}^{(m)}\right) & =q(i, j), \quad i, j=m, m+1, \ldots, \quad \text { and } \\
\lim _{m, k \rightarrow \infty} r\left(n_{i}^{(m)}, n_{i}^{(k)}\right) & =0 \text { for all } i .
\end{aligned}
$$

The convergence of the sequence $N_{i}=\left\{n_{i}^{(m)}\right\}_{m=i}^{\infty}$ in the space $(\mathcal{U}, \rho)$ as $m \rightarrow \infty$ to a point $u_{i} \in \mathcal{U}, i=1,2 \ldots$, follows from the fundamentality of the sequence, i.e., from the second equation; the first equation implies that the distance matrix of the limiting points $\left\{u_{i}\right\}$ coincides with $q$.

Let us now construct the sequences $N_{i}, i=1,2, \ldots$ We will construct the desired sequences $\left\{N_{i}\right\}_{i=1}^{\infty} \subset \mathbf{N}$ by induction.

Choose an arbitrary point $n_{1}^{(1)} \in \mathbf{N}$ and assume that for a given $m>1$, we have already determined the finite fragments $L_{k}=\left\{n_{i}^{(k)}\right\}_{i=1}^{k} \subset \mathbf{N}$ of the first $m$ sets $\left\{N_{i}\right\}_{i=1}^{m}$ for $k=1,2, \ldots, m$ such that $\max _{i, j=1, \ldots, k}\left|r\left(n_{i}^{(k)}, n_{j}^{(k)}\right)-q(i, j)\right|=\delta_{k}<2^{-k}, k=$ $1,2, \ldots, m$, and the sets $L_{k}$ are pairwise disjoint.

Our construction of the set $L_{m+1}$ depends only on the set $L_{m}$; thus for simplicity we can renumber the sets $L_{m}: n_{i}^{(m)}=i, i=1, \ldots, m$.

Let us construct a new set $L_{m+1}=\left\{n_{i}^{(m+1)}\right\}_{i=1}^{m+1} \subset \mathbf{N}$ with desired properties as follows. Consider a finite metric space $(V, d)$ consisting of $2 m+1$ points $y_{1}, \ldots, y_{m} ; z_{1}, \ldots, z_{m}, z_{m+1}$ with distances $d\left(y_{i}, y_{j}\right)=r_{i, j}, i, j=1, \ldots, m, d\left(z_{i}, z_{j}\right)=$ $q(i, j), i, j=1, \ldots, m+1 ; d\left(y_{i}, z_{j}\right)=q(i, j), i=1, \ldots, m, j=1, \ldots, m+1 ; i \neq j$, $d\left(y_{i}, z_{i}\right)=\delta_{m}, i=1, \ldots, m$, for some $\delta_{m}$. It is easy to check that these distances are well defined. Denote the distance matrix of the space $(V, d)$ by $q_{m}$. Apply Corollary 2 (on $\epsilon$-extension of isometries) and extend the set $L_{m}=\{1,2, \ldots, m\}$ with a new set $L_{m+1}$ consisting of $m+1$ positive integers $\left\{n_{i}^{(m+1)}\right\}_{i=m+1}^{2 m+1} \subset \mathbf{N}$ so that the distance matrix of the new space $L_{m+1}$ will differ from the NW-corner of order $m+1$ of the matrix $q$ by at most $\delta_{m}$, which is less than $2^{-(m+1)}: \max _{i, j}\left|r\left(n_{i}^{(m+1)}, n_{j}^{(m+1)}\right)-q_{m}(i, j)\right|=$ 
$\delta_{m+1}<2^{-(m+1)}$ (recall that the NW-corners of order $m$ of the matrices $q_{m}$ and $r$ coincide by construction). We see that for every $i$, the sequence $\left\{n^{\left(m_{i}\right)}\right\}_{m=i}^{\infty}$ is fundamental and $\lim _{m \rightarrow \infty} r\left(n_{i}^{(m)}, n_{j}^{(m)}\right)=q(i, j)$. Thus we have proved that every Polish space can be isometrically embedded in $\left(\mathcal{U}_{r}, \rho_{r}\right)$.

Now we can substantially strengthen Corollary 2:

Corollary 3 (on extension of isometries). The space $\left(\mathcal{U}_{r}, \rho_{r}\right)$ enjoys the following property: for every finite set $A=\left\{a_{i}\right\}_{i=1}^{n} \in \mathcal{U}_{r}$ and every distance matrix $q$ of order $N$, $N>n$, with $N W$-corner of order $n$ equal to the distance matrix $\left\{\rho\left(a_{i}, a_{j}\right)\right\}_{i, j=1}^{n}$, there exist points $a_{n+1}, \ldots, a_{N}$ such that the distance matrix of the whole set $\left\{a_{i}\right\}_{i=1}^{N}$ equals $q$.

The proof parallels that of Corollary 2 and uses the arguments given above.

Let us continue the proof of Theorem 3 .

2. In order to prove the homogeneity in the strong form 2'), take two arbitrary finite $n$-point isometric subsets $A=\left\{a_{i}\right\}_{i=1}^{n}$ and $B=\left\{b_{i}\right\}_{i=1}^{n}$ in $\left(\mathcal{U}_{r}, \rho_{r}\right)$ and choose an isometry between them by fixing the order of points corresponding to the isometry. Let us construct two isometric ordered countable subsets $C$ and $D$, each of them dense in $\mathcal{U}$, such that the first $n$ points of $C$ coincide with the points of $A$ in the chosen ordering, and the first points of $D$ coincide with the points of $B$. First we fix a countable everywhere dense subset $F$ in $\left(\mathcal{U}_{r}, \rho_{r}\right)$ such that $F \cap A=F \cap B=\emptyset$ and represent it as an increasing union: $F=\cup F_{n}$. Let $C_{1}=A \cup F_{1}$ and find a set $D_{1}=B \cup F_{1}^{\prime}$ such that the isometry of the subsets $A$ and $B$ extends to $F_{1}$ and $F_{1}^{\prime}$. Thus $D_{1}$ is isometric to $C_{1}$. This can be done by Corollary 4 (on extension of isometries).

Then choose $D_{2}=D_{1} \cup F_{2}$ and $C_{2}=C_{1} \cup F_{2}^{\prime}$ and again extend the isometry from the subsets $D_{1}$ and $C_{1}$ to the whole sets. Thus we have constructed an isometry between $D_{2}$ and $C_{2}$, etc. The alternating process yields two everywhere dense countable isometric sets $\cup C_{i}$ and $\cup D_{i}$, the isometry between them extending the isometry between the subsets $A$ and $B$. The method we have used is well known and is called in the literature the "back and forth" method; it was also used in Urysohn's paper.

3. Uniqueness. Let $r$ and $r^{\prime}$ be two universal proper distance matrices, and let $\left(\mathcal{U}_{r}, \rho_{r}\right)$ and $\left(\mathcal{U}_{r}^{\prime}, \rho_{r}^{\prime}\right)$ be the corresponding completions of the set of positive integers.

Let us construct in these spaces two countable everywhere dense subsets $F_{1}$ and $F_{2}$, respectively, so that the isometry between them extends to an isometry of the whole spaces. Denote by $\left\{x_{i}\right\}$ and $\left\{u_{i}\right\}$ the everywhere dense subsets of the spaces $\left(\mathcal{U}_{r}, \rho_{r}\right)$ and $\left(\mathcal{U}_{r}^{\prime}, \rho_{r}^{\prime}\right)$ that generate the matrices $r$ and $r^{\prime}$, respectively. Now we repeat the same argument as in the proof of the first claim of the theorem. We begin with a finite set of points $\left\{x_{i}\right\}_{i=1}^{n_{1}}$ in $\left(\mathcal{U}_{r}, \rho_{r}\right)$ and supplement it with a set of points $\left\{u_{i}^{\prime}\right\}_{i=1}^{m_{1}} \subset \mathcal{U}_{r}$ with the same distance matrix as that of the set $\left\{u_{i}\right\}_{i=1}^{m_{1}}$; this can be done by the universality of the space $\left(\mathcal{U}_{r}, \rho_{r}\right)$ (property 1 ), which is already proved. Now supplement the set $\left\{u_{i}\right\}_{i=1}^{m_{2}}\left(m_{2}>m_{1}\right)$ in the space $\mathcal{U}_{r}^{\prime}$ with a set of points $\left\{x_{i}^{\prime}\right\}_{i=1}^{n_{2}}\left(n_{2}>n_{1}\right)$ so that the distance matrix of the subset $\left\{u_{i}\right\}_{i=1}^{m_{1}} \cup\left\{x_{i}^{\prime}\right\}_{i=1}^{n_{1}}$ of the set $\left\{u_{i}\right\}_{i=1}^{m_{2}} \cup\left\{x_{i}^{\prime}\right\}_{i=1}^{n_{2}}$ will coincide with the distance matrix of the set $\left\{u_{i}^{\prime}\right\}_{i=1}^{m_{1}} \cup\left\{x_{i}\right\}_{i=1}^{n_{1}}$, etc; continuing this process to infinity, we obtain the desired two sets: $\left\{x_{i}\right\}_{i=1}^{\infty} \cup\left\{u_{i}^{\prime}\right\}_{i=1}^{\infty} \subset \mathcal{U}_{r}$ and $\left\{u_{i}\right\}_{i=1}^{\infty} \cup\left\{x_{i}^{\prime}\right\}_{i=1}^{\infty} \subset \mathcal{U}_{r}^{\prime}$, which are everywhere dense in their spaces and isometric. Thus we have completed the proof of the theorem. 
Theorems 1 and 3 yield the following remarkable fact:

Corollary 4 The generic distance matrix is a universal matrix; and hence the generic Polish space (in the sense of our model, i.e., the cone $\mathcal{R}$ ) is the Urysohn space $\mathcal{U}$.

In our terms, P. Urysohn first chooses a rational distance matrix, which determines the countable universal (noncomplete) metric space in the class of metrics with rational values, and its completion is the universal space $\mathcal{U}$. Urysohn (2]) observes that there exists a universal space of diameter 1 (or any given diameter). If we similarly define universal distance matrices with elements from the interval $[0,1]$, then the corresponding completion is precisely the universal space for Polish spaces of diameter 1, and all theorems of this section remain valid after trivial modifications. The question, posed in [2], whether there exist noncomplete universal (homogeneous) spaces was answered in the affirmative in [21]: it turns out that one can remove special subsets from the Urysohn space with preserving homogeneity, universality, and density of the remaining set. Katetov's construction of the universal space is slightly different from the original one and from our constructions; a close version was suggested by M. Gromov in [4] ${ }^{1}$.

Recently V. Uspensky [19] (see also 33]), using a theorem due to Torunczyk 25], proved, answering my question, that the Urysohn space is homeomorphic to the infinitedimensional Hilbert space (and hence to any separable Banach space). Although this result is hardly useful for constructing a realization of the Urysohn space, because the homeomorphism between the Urysohn space and the Hilbert space is highly nonconstructive and, which is most important, has no uniformness or smoothness, nevertheless it is of theoretical interest, because it shows that the Urysohn space can be regarded as a standard space in the infinite-dimensional topology, perhaps more natural than the Hilbert space. But the problem of constructing a more explicit realization of the Urysohn space, which was mentioned already by P. S. Alexandrov in his comments to the Russian translation of the main Urysohn's paper 2], is still actual: in contrast to, for example, illustrative models of the universal graph, we have no nonapproximative models of the Urysohn space.

In [19], Uspensky proved the universality of the group $\operatorname{Iso}(\mathcal{U})$ of isometries of the Urysohn space as a topological group in the compactly open topology: every Polish group can be continuously isomorphically embedded into $\operatorname{Iso}(\mathcal{U})$; the key point is the proof of the existence of an isometric embedding of an arbitrary Polish space into $\mathcal{U}$ such that its group of isometries is naturally isomorphically embedded into the group of isometries of the Urysohn space. V. Pestov [24] proved that the group $\operatorname{Iso}(\mathcal{U})$ has a fixed point property, i.e., every continuous action of this group on an arbitrary compact space has a fixed point. Among more special and not quite obvious properties of the Urysohn space, we mention that is is not isometric to its direct powers with natural metrics (F. Petrov) and that replacing its metric with any nonlinear concave function of the original metric also leads to spaces that are not isometric to the Urysohn space (i.e., are not universal). Thus the main open problems are as follows: to find a satisfactory model of the universal space and to describe the structure of the group of its

\footnotetext{
${ }^{1}$ It seems that the papers by Katetov [21] and Sierpinski [22] were the first ones, after many years, to deal with the Urysohn space; textbooks on measure-theoretic topology rarely make mention of it.
} 
isometries. In particular, the following special problem seems to be rather interesting: whether there exists an everywhere dense subgroup of the group of isometries that is the inductive limit of compact groups. A similar combinatorial problem (concerning the group of automorphisms of the universal graph) is recently solved, see the Appendix.

\section{The main theorem: universality of almost all distance matrices}

We will consider arbitrary Borel probability measures on the cone $\mathcal{R}$, or, in other words, arbitrary random metrics on the set of positive integers. Since the cone $\mathcal{R}$ is metrizable and separable in the weak topology, i.e., becomes a Polish space if we fix some metric compatible with the weak topology, it follows that the simplex of measures with the weak topology (topology of convergence on cylinder sets of the cone $\mathcal{R}$ ) is also a Polish space. This simplex is the inverse limit of the simplices of probability measures on the finite-dimensional cones $\mathcal{R}_{n}$.

Recall that in Section 2.3 we have constructed a family of measures $\nu_{\gamma}$ on the cone $\mathcal{R}_{n}$, indexed by an arbitrary measure $\gamma$ of full support on the half-line.

Theorem 4 The measures $\nu_{\gamma}$ constructed in Section 2.3 are concentrated on the set of universal matrices; in other words, almost every distance matrix with respect to any of these measures is universal. Therefore, the completion of the set of positive integers with respect to the metric determined by almost every matrix is isometric to the Urysohn universal space.

Proof We must check that $\nu_{\gamma}$-almost every matrix $r=\left\{r_{i, j}\right\}$ satisfies the universality condition. We strengthen this condition as follows: for every $k$, the empirical distribution of the column consisting of the first $k$ entries (for almost every given matrix) is of full support, i.e., the support of this empirical distribution coincides with the set $A\left(r^{k}\right)$. This is indeed a strengthening, since the universality condition requires only that the column vectors of length $k$ of a given matrix be dense in $A\left(r^{k}\right)$ and we will show that they appear with frequency corresponding to the given distribution on the set $A\left(r^{k}\right)$, which is of full support by construction. But, in view of Lemma 5 , the measure $\nu_{\gamma}$ is invariant and ergodic with respect to the shifts of the first $k$ rows for all $k$, and thus we can apply the ergodic theorem (= the law of large numbers), which asserts that the empirical distribution coincides with the theoretical distribution for almost all matrices, and the same lemma implies that the original distribution is of full support for every $k$.

Thus we have proved that $\nu_{\gamma}$-almost every distance matrix generates, as the completion of the set of positive integers with respect to the corresponding metric, the Urysohn space. These measures should be thought of as the most "dispersed" over the set of distance matrices; hence this theorem justifies the assertion that the random space is universal with probability one. Another paradoxical property of these measures will be considered in Section 5 .

However, we can assert much more. Denote by $\mathcal{V}$ the simplex of all Borel probability measures on the cone $\mathcal{R}$ and equip it with the weak topology, i.e., the topology of 
convergence on cylinder sets. Note that the set of nondegenerate (i.e., positive on nonempty open sets) measures is an everywhere dense $G_{\delta^{-}}$set in $\mathcal{V}$. The corollary of Theorems 1 and 3 immediately implies the following claim on genericity.

Theorem 5 The subset of measures on $\mathcal{V}$ concentrated on universal matrices is an everywhere dense $G_{\delta}$-set in $\mathcal{V}$. Thus, with respect to the generic measure $\nu$ on $\mathcal{R}, \nu$ almost every distance matrix is universal and hence determines a metric $r$ on the set of positive integers $\mathbf{N}$ such that the completion of $\mathbf{N}$ with respect to this metric is the Urysohn space.

This assertion follows immediately from the general fact that the set of all measures defined on a Polish space and concentrated on a fixed everywhere dense $G_{\delta^{-}}$subset is itself an everywhere dense $G_{\delta}$-subset of the space of all measures in the weak topology. Thus we have another confirmation of the assertion that the Urysohn space is generic, now in the probabilistic sense: the random countable metric space is isometric to an everywhere dense subset of the Urysohn space, or, in other words, the completion of the random countable metric space is the Urysohn space with probability one; the "randomness" is understood with respect to any measure from a $G_{\delta}$-subset. However, this assertion, unlike the theorem proved above, yields no interesting concrete examples of such measures (as the measures $\nu_{\gamma}$ ). In the next section we will study measures on the cone of distance matrices in more detail.

\section{Matrix distributions and a generalization of Kolmogorov's problem of extension of measures}

\subsection{Invariants of metric triples, and the uniqueness theo- rem}

We will consider metric spaces with measure and random metrics on the set of positive integers. Assume that $(X, \rho, \mu)$ is a Polish space with metric $\rho$ and Borel probability measure $\mu$. We say that $(X, \rho, \mu)$ is a metric triple (in [4, the term "mm-space" = metric-measure space is used; other terms are "probability metric space," "Gromov triple"). Two metric triples $\left(X_{1}, \rho_{1}, \mu_{1}\right)$ and $\left(X_{2}, \rho_{2}, \mu_{2}\right)$ are isomorphic if there exists a measure-preserving isometry $V$ :

$$
\rho_{2}(V x, V y)=\rho_{2}(x, y), \quad V \mu_{1}=\mu_{2}
$$

As we have already mentioned, the classification of (noncompact) Polish spaces is a "wild" problem. It is surprising that the classification of metric triples is a "tame" problem and has a reasonable answer in terms of the action of the infinite group of permutations $S_{\infty}$ and $S_{\infty}$-invariant measures on $\mathcal{R}$.

Given a metric triple $T=(X, \rho, \mu)$, consider its infinite product with the Bernoulli measure $\left(X^{\mathbf{N}}, \mu^{\mathbf{N}}\right)$ and define a mapping $F: X^{\mathbf{N}} \rightarrow \mathcal{R}$ as follows:

$$
F_{T}\left(\left\{x_{i}\right\}_{i=1}^{\infty}\right)=\left\{\rho\left(x_{i}, x_{j}\right)\right\}_{i, j=1}^{\infty} \in \mathcal{R}
$$


The $F_{T}$-image of the measure $\mu^{\mathbf{N}}$, which we denote by $D_{T}$, is called the matrix distribution of the metric triple T: $F_{T} \mu^{\infty} \equiv D_{T}$.

The group $S_{\infty}$ of all finite permutations of positive integers (= the infinite symmetric group $S_{\mathbf{N}}$ ) acts both on $\mathbf{M}_{\mathbf{N}}(\mathbf{R})$ and on the cone $\mathcal{R}$ of distance matrices as the group of simultaneous permutations of rows and columns of matrices.

Lemma 9 The measure $D_{T}$ is a Borel measure on $\mathcal{R}$, invariant and ergodic with respect to the action of the infinite symmetric group and the action of the $N W$-shift (the simultaneous shift of an infinite matrix in the vertical (up) and horizontal (to the left) direction: $\left.(N W(r))_{i, j}=r_{i+1, j+1} ; i, j=1,2, \ldots\right)$.

Proof All claims follow from the analogous properties of the measure $\mu^{\infty}$, which is invariant under the shift and permutations of coordinates, and from the fact that the mapping $F_{T}$ commutes with the action of the shift and permutations.

We say that a measure on a metric space is nondegenerate if every nonempty open set has a positive measure.

Theorem 6 Two metric triples $T_{1}=\left(X_{1}, \rho_{1}, \mu_{1}\right)$ and $T_{2}=\left(X_{2}, \rho_{2}, \mu_{2}\right)$ with nondegenerate measures are equivalent if and only if their matrix distributions coincide as measures on the cone $\mathcal{R}: D_{T_{1}}=D_{T_{2}}$.

Proof It is obvious that the matrix distributions of equivalent triples coincide: if there is a measure-preserving isometry $V: X_{1} \rightarrow X_{2}$ between $T_{1}$ and $T_{2}$, then its infinite power $V^{\infty}$ preserves the Bernoulli measure: $V^{\infty}\left(\mu_{1}^{\infty}\right)=\mu_{2}^{\infty}$; hence, since $F_{T_{2}} X_{2}^{\infty}=F_{T_{2}}\left(V^{\infty} X_{1}^{\infty}\right)$, the images of these measures coincide: $D_{T_{2}}=D_{T_{1}}$.

Now assume that $D_{T_{2}}=D_{T_{1}}=D$. Then $D$-almost all distance matrices $r$ are the images, under the mappings $F_{T_{1}}$ and $F_{T_{2}}$, of some sequences, say $r_{i, j}=\rho_{1}\left(x_{i}, x_{j}\right)=$ $\rho_{2}\left(y_{i}, y_{j}\right)$; but this means that identifying $x_{i} \in X_{1}$ and $y_{i} \in X_{2}$ for all $i$ is an isometry $V$ between these countable sets. Since the measures are nondegenerate, these sequences are everywhere dense with probability one; hence the isometry extends to the whole image and preimage. Let us prove that this isometry preserves the measure. This is the key point: by the ergodic theorem, $\mu_{1}$-almost all sequences $\left\{x_{i}\right\}$ and $\mu_{2}$-almost all sequences $\left\{y_{i}\right\}$ are uniformly distributed on $X_{1}$ and $X_{2}$, respectively. This means that our sequences can be chosen so that the $\mu_{1}$-measure of every ball of rational radius centered at the points of our sequences $B^{l}\left(x_{i}\right) \equiv\left\{z \in X_{1}: \rho_{1}\left(x_{i}, z\right)<l\right\}$ will equal

$$
\mu_{1}\left(B^{l}\left(x_{i}\right)\right)=\lim _{n \rightarrow \infty} \frac{1}{n} \sum_{k=1}^{n} 1_{[0, l]}\left(\rho_{1}\left(x_{k}, x_{i}\right)\right)
$$

(countably many conditions). But since $V$ is isometric (because $r_{i, j}=\rho_{1}\left(x_{i}, x_{j}\right)=$ $\rho_{2}\left(y_{i}, y_{j}\right)$, see above), the same expression is the $\mu_{2}$-measure of the ball $B^{l}\left(y_{i}\right) \equiv\{u \in$ $\left.X_{2}: \rho_{2}\left(y_{i}, u\right)<l\right\}$ :

$$
\mu_{2}\left(B^{l}\left(y_{i}\right)\right)=\lim _{n \rightarrow \infty} \frac{1}{n} \sum_{k=1}^{n} 1_{[0, l]}\left(\rho_{2}\left(y_{k}, y_{i}\right)\right)=\mu_{1}\left(B^{l}\left(x_{i}\right)\right) .
$$


Finally, since the measures are nondegenerate and hence, as mentioned above, the sequences $\left\{x_{i}\right\}$ and $\left\{y_{i}\right\}$ are everywhere dense each in its space, it follows that if the values of two (Borel) measures on a countable set of balls with rational radii coincides, then the $V$-image of the measure in the first space coincides with the measure in the second space.

Corollary 5 The matrix distribution is a complete invariant of equivalence classes (up to measure-preserving isometry) of metric triples with nondegenerate measures.

We call this result the "uniqueness theorem," because it asserts that a metric triple with a given matrix distribution is unique up to equivalence. This theorem, in another form and under the name of "Reconstruction Theorem," was originally proved in the book [4, p. 117-123]. The theorem was stated in terms of finite-dimensional distributions of the measure that we call the matrix distribution, and its proof involved analytical techniques. The "ergodic" proof given above was the answer to the question posed by Gromov in 1997: how one can avoid complicated arguments. This proof was also given in [5] and cited in the book [4, p. 122-123]. The same book invites the reader to compare both proofs and explain how the ergodic theorem replaces analytic arguments (such as the Weierstrass theorem and the method of moments). The explanation is as follows: the ergodic theorem allows one to use, instead of approximative techniques, an infinite (limiting) object (infinite orbits, measures on the limiting space, etc.) and its properties (for example, equidistribution) that cannot even be formulated for finite objects. In our case, considering infinite matrices and the cone $\mathcal{R}$ with an invariant measure allows us to reduce the problem to studying an ergodic action of the infinite symmetric group. In [6, 8] we use this ergodic machinery to obtain a much more general result - the classification of measurable function of several arguments; above we have considered a special case of this problem: a metric is a function of two arguments on a metric space with measure.

\subsection{Existence theorem}

By definition, the matrix distribution of a metric triple $T=(X, \rho, \mu)$ with a nondegenerate measure $\mu$ is a measure $D_{T}$ on the cone $\mathcal{R}$. In this section we consider random matrices (or measures on $\mathcal{R}$ ) that can be matrix distributions; in other words, we want to describe those distributions on distance matrices that arise as the matrix distributions of a sequence of independently chosen points $\left\{x_{i}\right\}$ of some metric space $(X, \rho)$ distributed according to some measure $\mu$ on this space. To obtain such a characterization is also necessary in order to assert that the classification problem is indeed "smooth," i.e., that the set of invariants is described explicitly. We will show that the set of matrix distributions is a Borel subset of the space of all probability measures on the cone $\mathcal{R}$.

As mentioned above (Lemma 7), every measure $D_{T}$ must be invariant and ergodic under the action of the symmetric group and NW-shift. But this condition is not sufficient, and there are additional conditions. We give necessary and sufficient conditions on the measure (see also $[\underline{8}$ ); below we present counterexamples that show that these conditions are substantial. 
Theorem 7 (Existence of a metric triple with a given matrix distribution).

Let $D$ be a Borel probability measure on the cone of distance matrices $\mathcal{R}$ that is invariant and ergodic under the action of the infinite symmetric group.

1) The following condition is necessary and sufficient for the measure $D$ to be the matrix distribution of some metric triple $T=(X, \rho, \mu)$ :

for every $\epsilon>0$, there exists an integer $N=N(\epsilon)$ such that

$$
D\left\{r=\left\{r_{i, j}\right\} \in \mathcal{R}: \lim _{n \rightarrow \infty} \frac{\left|\left\{j: 1 \leq j \leq n, \min _{1 \leq i \leq N} r_{i, j}<\epsilon\right\}\right|}{n}>1-\epsilon\right\}>1-\epsilon .
$$

2) The following stronger condition is necessary and sufficient for the measure $D$ to be the matrix distribution of some metric triple $T=(X, \rho, \mu)$ with a compact metric space $(X, \rho)$ :

for every $\epsilon>0$, there exists an integer $N=N(\epsilon)$ such that

$$
D\left\{r=\left\{r_{i, j}\right\} \in \mathcal{R}: \min _{1 \leq i \leq N} r_{i, j}<\epsilon \text { for all } j>N\right\}>1-\epsilon .
$$

Here we will only sketch the proof of this theorem (see $[6$ for details).

A. Necessity. In the case of a compact space the necessity is obvious: condition (5) means that a sufficiently long sequence of independent points of the space distributed according to the measure $\mu$ contains an $\epsilon$-net of the compact metric space for every $\epsilon$. In the general case, the necessity of condition (4) follows automatically from the following well-known property of Borel measures in Polish spaces: there is a sigma-compact set of full measure (the so-called "regularity" of measures); therefore for every $\epsilon>0$, there exists a compact set of measure $>1-\epsilon$. Hence, by the countable additivity of the measure, for every $\epsilon>0$, there exists finitely many points such that the measure of the union of the $\epsilon$-balls centered at these points is greater than $1-\epsilon$; using the ergodic theorem, we can assert that the condition inside the braces in (4) is satisfied for distance matrices from a set of measure greater than $1-\epsilon$.

B. Sufficiency. Now assume that $D$ is an invariant and ergodic measure on the cone $\mathcal{R}$ satisfying condition (4). Choose an arbitrary $D$-generic distance matrix - one can check that $D$-almost all matrices satisfy the desired conditions - and construct a complete metric space by completing the set of positive integers with respect to the corresponding metric; the set of positive integers forms an everywhere dense family in this space. Using the classical law of large numbers, one can easily find the values of the measure on the set algebra generated by the balls of arbitrary radii centered at the chosen points, and condition (4) allows one to prove that this measure extends to a countably additive Borel measure. Then one checks that the matrix distribution of the obtained metric triple coincides with the measure $D$.

Remark The structure of the necessary and sufficient conditions on the measure in the above theorem shows that matrix distributions form a Borel subset in the set of all Borel probability measures on the cone $\mathcal{R}$. Thus the classification of metric triples is a smooth (Borel) classification, because the space of types is a Borel set of matrix distributions in the set of Borel probability measures on the cone of distance matrices. The same conclusion holds for a more general problem of classification of measurable functions of several arguments, see [8]. 
Condition (4) can be replaced with another condition from the paper [8], the socalled "simplicity" of an $S_{\infty}$-invariant (or symmetric) measure, which is stated below. This condition guarantees that a measure $D$ is the matrix distribution of a measurable function of two variables, and in view of the uniqueness theorem, this suffices for our purposes. It remains to check that the matrix distribution of the constructed metric triple coincides with the original measure.

\subsection{Uniform distribution of sequences in metric spaces and a generalization of Kolmogorov's problem on extension of measures}

Let us turn to a more detailed analysis of measures on the cone of distance matrices and properties of individual matrices.

Let $(X, \rho)$ be a metric space, and let $x=\left\{x_{i}\right\}_{i=1}^{\infty}$ be an everywhere dense sequence of points in $X$. We say that this sequence is regular if for every finite $n$ and every open subset $C \subset R^{n}$, the limit

$$
\lim _{n \rightarrow \infty} \frac{1}{n} \sum_{k=1}^{n} 1_{C}\left(\left\{\rho\left(x_{i}, y_{k}\right)\right\}_{i=1}^{n}\right)
$$

exists. In particular, for every positive integer $i$ and every positive $r$, the limits

$$
\left.\mu\left(D_{r}\left(x_{i}\right)\right) \equiv \lim _{n \rightarrow \infty} \frac{1}{n} \sum_{k=1}^{n} 1_{[0, r)}\left(\rho\left(x_{i}, y_{k}\right)\right\}\right)
$$

exist.

The latter condition is a specialization of the former one; it means, in particular, that one can find the "empirical" measures of open balls $D_{r}\left(x_{i}\right)$ of radius $r$ centered at the points $x_{i}$ of our sequence; and the more general first condition allows one to find the joint distribution of the distances to an arbitrary finite number of points of our sequence, i.e., to find the measures of intersections and unions of balls, etc.; in other words, this condition allows one to determine a finitely additive measure $\mu_{x}$ on the set algebra of the space $X$ generated by the open balls centered at the points of the sequence. One may say that a regular sequence is uniformly distributed with respect to the measure $\mu_{x}$ determined by it. If we were given a Borel probability (countably additive) measure $\bar{\mu}$ on the space $(X, \rho)$, then the classical law of large numbers would imply that a realization of the sequence of i.i.d. points of $X$ distributed according to the measure $\mu$ is regular with probability one, and the above conditions show how one can recover the measure. But if the measure is not given a priori, the question arises: whether for every regular sequence $x=\left\{x_{i}\right\}, i=1,2, \ldots$, the finitely additive measure $\mu_{x}$ extends to a Borel probability measure $\bar{\mu}$ on the space $(X, \rho)$ ? In other words, whether every regular sequence is uniformly distributed with respect to some probability measure?

This question is similar to the questions that arise in connection with the classical Kolmogorov theorem on extension of measures and its generalizations: in that setting, 
given a measure defined on the algebra of cylinder sets of a vector space, one checks that it is countably additive on the algebra of cylinder sets, and then this measure extends to a true probability measure on the $\sigma$-algebra. By the Kolmogorov theorem, this extension always exists in the linear space $R^{\infty}$, but in other spaces this is true not for all cylinder measures. More precisely, our analogy with the Kolmogorov theorem is as follows.

Consider the mapping $\Pi \equiv \Pi_{x}: X \rightarrow \mathbb{R}_{+}^{\infty}$ that associates with a point $y$ of the space $X$ the sequence of its distances $\left\{\rho\left(x_{i}, y\right)\right\}, i=1,2, \ldots$, to the points of the dense set $x=\left\{x_{i}\right\}$. Obviously, this mapping sends closed balls centered at $x_{i}$ to cylinder sets, and the set algebra generated by these balls to a subalgebra of the algebra of cylinder sets of the space $\mathbb{R}_{+}^{\infty}$. Since the sequence $x=\left\{x_{i}\right\}_{i}$ is everywhere dense, the mapping $\Pi_{x}$ is monomorphic. As mentioned above, the regularity of $x$ allows us to find the joint distributions of coordinates in the image, and thus a cylinder measure in $\mathbb{R}_{+}^{\infty}$; by the Kolmogorov theorem, this measure extends to a probability (countably additive) measure $\tau \equiv \tau_{x}$ in $\mathbb{R}_{+}^{\infty}$. However, this does not mean that the mapping $\Pi_{x}$ is an isomorphism of measure spaces; it is only a homomorphism of a space with finitely additive measure to a space with probability measure. Moreover, a measure on the algebra generated by the balls centered at the points of an everywhere dense sequence does not always extend to a countably additive measure on the $\sigma$-algebra; namely, the following assertion holds.

Lemma 10 A finitely additive measure $\mu_{x}$ extends to a Borel probability measure $\bar{\mu}_{x}$ in the space $(X, \rho)$ if and only if the $\tau_{x}$-measure of $\Pi_{x}(X)$ is equal to one.

Condition (4) (or the simplicity condition, see below) guarantees that the extension exists; in general, if this condition is not satisfied, the measure is not countably additive: in the general case, the measure $\tau_{x}\left(\Pi_{x}(X)\right)$ may be equal to zero, as we will see later. In this case it is natural to ask whether we can extend the space $(X, \rho)$ so that the measure will equal to one; I have not a complete answer to this question, but in some cases such a compactification of the space is possible. However, in this problem we deal with a "nonlinear" version of Kolmogorov's extension problem, and there hardly exists an exact analog of the unified space $\mathbb{R}^{\infty}$ in which the Kolmogorov theorem holds for any cylinder measure.

Example 1 A trivial example when there is no countable additivity is as follows. Let $(X, \rho)$ be a countable set of points with unit pairwise distances, and let $x$ be simply the sequence of all points of the space $X$. This sequence is obviously regular, because its distance matrix is the matrix with units outside the diagonal; hence the measure $\tau$ - the joint distribution of distances - is the $\delta$-measure at the point $(1,1, \ldots) \in \mathbb{R}_{+}^{\infty}$. And on the space $(X, \rho)$ there is only a finitely additive measure $\mu$, which vanishes on finite sets and equals one on cofinite sets.

Before considering more serious examples, let us formulate the question in terms of measures on the cone of distance matrices and their symmetrizations. The definition of regularity immediately implies the following result.

Lemma 11 It is necessary and sufficient for a matrix $r \in \mathcal{R}$ to be the distance matrix of a regular sequence of some metric space that there exist the empirical distribution 
of the rows (columns) of $r$ as a probability measure in the space $\mathbb{R}^{\infty}$. This measure coincides with the measure $\tau$ defined above.

We will call such distance matrices regular and denote by $\Psi$ the mapping from the set of regular matrices to the set of probability measures in the space $\mathbb{R}^{\infty}$ that associates with a regular matrix the empirical distribution of its rows.

Without dwelling on details, we note that every regular distance matrix generates, by the symmetrization

$$
\bar{\mu}_{B}=\lim (n !)^{-1} \sum_{g \in S_{n}} 1_{B}(g r)
$$

an $S_{\infty}$-invariant measure on the cone of distance matrices. Here $B$ is a cylinder set in the space of matrices, and an element $g$ acts on a matrix $r$ by a simultaneous permutation of rows and columns.

Theorem 8 The following assertions are equivalent:

1. A regular everywhere dense sequence $x=\left\{x_{i}\right\}$ of a Polish space $(X, \rho)$ is uniformly distributed with respect to some Borel probability measure $\mu$ (of full support) on the space $(X, \rho)$.

2. The symmetrization of the regular distance matrix $\left\{\rho\left(x_{i}, x_{j}\right)\right\}$ is a matrix distribution.

The matrix distribution from assertion 2 is precisely the invariant of the metric triple $(X, \rho, \mu)$ in the sense of Theorem 6 .

Thus we have two equivalent questions:

1. When a regular sequence of points of a Polish space is uniformly distributed with respect to some Borel (countably additive) probability measure in this space?

2. When a symmetric ergodic measure on the cone of distance matrices is the matrix distribution of a metric triple?

The answer to the first question is given by the lemma, and the answer to the second question is given by condition (4) or a more general condition of simplicity of a measure on the space of matrices:

A symmetric (i.e., $S_{\infty}$-invariant) measure $\lambda$ on the cone of distance matrices $\mathcal{R}$ is called simple if the mapping $\Psi$ is an isomorphism of measure spaces $(\mathcal{R}, \lambda)$ and $\left(\mathbb{R}^{\infty}, \Psi(\lambda)\right)$; in other words, $\lambda$-almost every distance matrix is uniquely determined by the empirical distribution of its rows (columns). The notion of simplicity is introduced in [8] and makes sense for arbitrary measures on spaces of matrices.

Theorem 9 (see [6]). A symmetric ergodic measure on the cone $\mathcal{R}$ is a matrix distribution if and only if it is simple.

Sometimes it is rather difficult to check the simplicity condition; however, it is obvious, for example, that a product measure on the space of matrices is not simple.

Example 2 First note that any symmetric matrix with zeros on the main diagonal and entries $r_{i, j}, i \neq j$, taking values in the interval $[1 / 2,1]$ is a proper distance matrix, because in this case the triangle inequality holds automatically for any triple of indices. Hence every probability measure $m$ supported by the interval $[1 / 2,1]$ generates the 
product measure $m^{\infty}$ concentrated on the cone $\mathcal{R}$; this means that all above-diagonal entries of the matrix are independent with respect to the measure $m^{\infty}$ and identically distributed with distribution $m$. Obviously, the measure $m^{\infty}$ is invariant and ergodic under the action of the group of permutations; if $m$ is not a $\delta$-measure, then $m^{\infty}$ is a continuous (nondiscrete) measure. The law of large numbers implies that almost every matrix is regular. Since nondiagonal elements (pairwise distances) in this example are bounded away from zero, almost every matrix determines a discrete metric space, which cannot have a continuous measure. Hence the measure $m^{\infty}$ is not the matrix distribution of any metric triple. It is equally obvious that the measure $m^{\infty}$ is not simple. One easily computes that in this example the measure $\tau_{x}\left(\Pi_{x}(X)\right)$ vanishes.

However, it is not difficult to prove that this is the unique (up to replacing the interval $[1 / 2,1]$ with any interval of the form $[a / 2, a], a>0$ ) class of examples when the entries of the random distance matrix can be independent random variables. Example 1 given above is a degenerate case: $m=\delta_{1}$.

Let us say that a regular sequence $x=\left\{x_{i}\right\}$ in a metric space is completely regular if any of the following equivalent conditions holds:

a) the measure $\mu_{x}$ extends to a countably additive measure, and hence the sequence $x$ is uniformly distributed with respect to this measure;

b) the symmetrization of the distance matrix of the sequence $x$ generates a matrix distribution;

c) the $\tau_{x}$-measure of the image $\Pi_{x}(X)$ is equal to one.

A regular sequence such that there is no countably additive measure with respect to which it is uniformly distributed will be called anarchical. It is easy to prove that in a locally compact metric space (which means in this context that every closed ball is compact) there are no anarchical sequences.

The above examples show that in discrete metric spaces, anarchical sequences are simply sequences consisting of all points of the space.

A much more surprising example is given by the Urysohn space.

Proposition 2 1. Consider the measure $\nu_{\gamma}$ constructed in Section 3; its symmetrization is not a matrix distribution.

2. Almost every matrix with respect to this measure determines an anarchical sequence in the Urysohn space. Thus this sequence is not uniformly distributed with respect to any probability measure in this space.

This result was discovered after O. Bohigos, Ch. Schmidt, and E. Bogomolny at my request had made experiments on computing the several first distributions of the symmetrization of the measures $\nu_{\gamma}$ and their spectra. Their computations showed that the spectrum of the random distance matrix in this case is governed by the Wigner semicircle law and that, for example, the support of the distribution of the symmetrized matrix entry is bounded away from zero. This means precisely that the symmetrized measure is not the matrix distribution of any metric triple with a nondiscrete space the result that we managed to prove. The underlying reason for this phenomenon is that the symmetrization of a measure concentrated on universal matrices may no longer be concentrated on them. This is the case with the measure $\nu_{\gamma}$. Thus the "most random" distance matrices determine no measure on the Urysohn space; they are "too random" 
and in a certain sense correspond to the "white noise" in this space. It is not known whether this means that there exists a virtual metric space (say, some compactification) in which these finitely additive measures extend to a probability measure. However, this effect emphasizes the similarity between the Urysohn space and the universal graph (see the Appendix). In order to construct probability measures in this space, we need universal matrices of more special form. We will return to this question elsewhere.

\subsection{Measure-theoretic space of metric triples}

We sought for invariants of metric triples as invariants of a measurable function of two variables, i.e., invariants of the metric regarded as a symmetric function on the direct square of the metric space with the product measure. Since a Polish space with a Borel continuous probability measure is isomorphic, as a measure space, to the interval $[0,1]$ with the Lebesgue measure, the problem reduces to classification of symmetric measurable functions of two variables. In fact, instead of the ordinary point of view, when one considers the set of all Borel measures on a given topological or metric space, we, on the contrary, fix the structure of a measure space (Lebesgue space) and consider all measurable (semi)metrics on this space (see [10, §6]). Let us consider this point of view in more detail.

Assume that $(X, \mu)$ is a Lebesgue space with a finite or $\sigma$-finite measure $\mu$ (for example, the interval $[0,1]$ or the line with the Lebesgue measure) and $S_{\mu}(X)$ is the space of all mod0 classes of measurable functions on $(X, \mu)$; define the cone $\mathcal{R}^{c} \subset$ $S_{\mu}(X)$ of measurable metrics, i.e., the cone of classes of mod0 coinciding symmetric functions of two arguments $\rho:(X \times X, \mu \times \mu) \rightarrow \mathbf{R}_{+}$satisfying the inequality

$$
\rho(x, y)+\rho(y, z) \geq \rho(x, z) \text { for }(\mu \times \mu \times \mu) \text {-almost all }(x, y, z) \in(X \times X \times X) .
$$

It is also natural to assume that $(\mu \times \mu)\{(x, y): \rho(x, y)=0\}=0$. In order to single out true metrics, impose on $\rho$ the following purity condition $\left([]_{0}\right)$ : the partition of $(X, \mu)$ into the classes $x \sim y \Leftrightarrow \rho(x, \cdot)=\rho(y, \cdot) \bmod 0$ is the partition into points mod0.

Functions satisfying all these conditions will be called almost metrics. It is essential that $\rho$ is not an individual function, but a class of $\bmod 0$ coinciding functions, hence $a$ priori it is not obvious that there exists an individual function (lifting) that determines the structure of a metric space on $X$ in the literal sense. If $X$ is a countable or finite set with counting measure, then we obtain the cone $\mathcal{R}$ or $\mathcal{R}_{n}$ of distance matrices from Section 2. Thus the cone $\mathcal{R}^{c}$ is a continuous generalization of the cone $\mathcal{R}$ to the case of a continuous measure.

Now assume that the measure $\mu$ is finite and $\rho \in \mathcal{R}^{c}$ is an almost metric; let a measure $D_{\rho}$ on the space $\mathbf{M}_{\infty}(\mathbf{R})$ be the matrix distribution of the measurable function $\rho$ (see the definition in the previous section). It follows from the ergodic theorem that $D_{\rho}\left(r \in \mathcal{R}: r_{i, k}+r_{j, k} \geq r_{i, k}\right)=1$ for any $i, j, k \in \mathbf{N}$, and hence $D_{\rho}(\mathcal{R})=1$. Using the characterization of matrix distributions given in Section 4, we obtain the following assertion.

Proposition 3 The measure $D_{\rho}$ is concentrated on the cone $\mathcal{R}$ (i.e., $D_{\rho}(\mathcal{R})=1$ )

and is an ergodic $S_{\infty}$-invariant measure. Therefore, every almost metric $\rho \in \mathcal{R}^{c}$ on $(X \times X, \mu \times \mu)$ determines a mod0 metric on the space $(X, \mu)$. 
Corollary 6 The set of classes of mod 0 coinciding measurable semimetrics on a Lebesgue space with continuous measure coincides with the set of classes of mod0 coinciding metric triples with continuous (finite) measure.

\section{Appendix: examples of other categories where randomness and universality coincide}

"Consider some total and hence unique copy of $A . "$ P. O. di Bartini

Soviet Math. Dokl., vol. 163, no. 4, p. 861-864 (1965).

\section{General setting. Universal and random graphs}

Consider a category in which there is a universally attracting object, i.e., an object such that every other object is "isomorphic" to its subobject. Assume that at the same time we are given a definition of the random object in the category; for example, this is possible if the objects of the category can be determined by finite or countable collections of numbers ("structural constants") satisfying certain conditions, and random objects are determined by random, in the ordinary sense, collections of numbers, i.e., by collections of random structural constants subject to certain conditions. In this case we can pose the following question: what objects will appear with probability one, and whether they will be almost always isomorphic to the universal object. Of course, the definition of randomness must satisfy certain requirements, which can be specified following the above pattern. ${ }^{2}$

Within this extremely general setting, which should be specified (this will be done elsewhere), one may say that the case of the category of Polish spaces gives the positive answer to the question posed above. But it will undoubtedly remind one of close situations in other categories, and first of all an analogy with the Erdös-Rényi theorem [15] on random and universal graphs, which asserts that the random graph is universal with probability one; see definitions below and in [14, 16. This simple theorem is also the simplest case of the above scheme, because every graph generates a metric space with distance metric on the set of vertices, and in the case of the universal graph, the distances between distinct points assume only two values: 1 and 2 . Hence (see the example in Section 2.3) the triangle inequality is automatically satisfied. It is natural to pursue the analogy with metric spaces by using distance matrices; but since a graph is usually determined by its adjacency matrix (which can be uniquely recovered from the distance matrix of the graph and uniquely determines it), we will also follow this tradition. More precisely, the random graph in the sense of [15] is given by a random adjacency matrix (symmetric $(0,1)$-matrix) with i.i.d. entries with

\footnotetext{
${ }^{2}$ Here it seems especially useful to use Kolmogorov's complexity-based definition of randomness.
} 
probabilities $(p, 1-p)$, where $0<p<1$. Then the distance matrix, which is a $(1,2)$ matrix, also has a similar distribution. On the other hand, the universality of a graph $\Gamma$ in the sense of 14 means that

1) any finite graph is isomorphic to a subgraph of $\Gamma$;

2 ) for every $n$, the group of automorphisms of the graph $\Gamma$ acts transitively on the set of all subgraphs of $\Gamma$ of order $n$ isomorphic to an arbitrary given graph.

Using the "back and forth" method, one can easily prove that such a graph is unique up to isomorphism; see, e.g., [16]. The similarity with the situation considered in this paper becomes especially strong if we compare the universality condition for graphs with the universality criterion for metric spaces (Theorem 1). Namely, the following result holds.

Let us say that an infinite symmetric $(0,1)$-matrix $\left\{a_{i, j}\right\}$ with zero diagonal is universal in the class of $(0,1)$-matrices if for every $n$ and every word $w_{1} w_{2} \ldots w_{n}$ of length $n$ in the alphabet $\{0,1\}$, there exists a number $k, k>n$, such that $a_{i, k}=w_{i}, i=$ $1,2, \ldots, k$, i.e., this word occurs as the beginning of the $k$ th column of the matrix. This definition is the discrete version of the definition of universality of distance matrices.

Lemma 12 A countable graph is universal if and only if its adjacency matrix is universal.

The proof follows immediately from the definition. (Cf. [16, where the universality criterion for graphs is stated in a slightly different form.) The set of universal matrices is an everywhere dense $G_{\delta}$-set in the set of all symmetric $(0,1)$-matrices with zero diagonal in the natural totally disconnected topology.

But, on the other hand, almost all random graphs in the Erdös-Rényi sense obviously satisfy this universality condition, because all their above-diagonal entries are independent; moreover, it is easy to deduce from this criterion that there are many other measures on the space of adjacency matrices with respect to which almost all graphs are universal; it is sufficient that almost all matrices contain all binary words as beginnings of their columns. Such measures also form an everywhere dense $G_{\delta^{-}}$set in the space of adjacency matrices (or distance matrices) in the weak topology; Bernoulli measures are only an interval in this large set.

Thus in the category of graphs there are exact analogs of all facts proved in this paper for the category of Polish spaces; one may say that the theory of universal and random graphs is an exact elementary model of the theory of universal and random metric spaces. Note that in the literature on graphs, the term "random graph" traditionally means "universal graph"; although this usage of terms is motivated by the Erdös-Rényi theorem, it contradicts the meaning of the notion of randomness, because randomness can be understood in different ways, but universality is uniquely understood and may take place for almost all matrices.

In this connection, let us mention another feature of the theory of universal graphs, which distinguish them from the Urysohn space. Since the universal graph is unique up to isomorphism, any two universal adjacency matrices lie on the same orbit of the infinite symmetric group, which acts naturally on the vertices of the graph (or by simultaneous permutations of the rows and columns of matrices). Hence the group of all permutations acts transitively on the set of universal matrices, and we obtain 
another example of the paradoxical situation first observed by Kolmogorov (see [39]): a group that acts transitively has a continuum of pairwise singular invariant ergodic measures.

The group of all automorphisms of the universal graph, as well as the group of all isometries of the Urysohn space, is of extreme interest; we will return to this subject later. Now we only mention that the recent paper [40] gave the positive answer to the question on existence of a dense locally finite subgroup in the group of automorphisms of the universal graph. (Compare with the similar question for the Urysohn space at the end of Section 3.)

The considered example is a very special case ("toy model") of the universality of metric spaces studied in this paper; in this case we have only one additional condition: the metric assumes only two nonzero values: 1 and 2. Indeed, consider the set of proper distance matrices whose nondiagonal entries equal 1 or 2 . In this case the triangle inequality is automatically satisfied for all symmetric $(1,2)$-matrices. One can define the notion of universality of such matrices by analogy with the definition from Section 3: every finite sequence of 1's and 2's occurs as the beginning of some column of the distance matrix. On the other hand, every graph determines a (geodesic) metric on the set of vertices.

Lemma 13 The set of universal distance (1,2)-matrices coincides with the set of distance matrices of universal graphs.

Thus the universal graph, regarded as a metric space, is the universal (and homogeneous) space in the class of countable metric spaces with metrics assuming two nonzero values: 1 and 2 .

In a similar way one can consider universal metric spaces with metrics assuming finitely many values.

\section{Several further examples and comments}

Let us briefly mention examples of other categories in which a similar situation takes place.

1. Universal and random simplices.

Consider the category of separable compact simplices. It is easy to construct a simplex whose set of extreme points is everywhere dense - the Poulsen simplex P. It is less known that this simplex is universal: every compact simplex can be affinely continuously embedded in $\mathrm{P}$ as a face, and any two isomorphic faces can be sent to each other by an affine isomorphism of the whole simplex ([18, 17]). Note that the set of extreme points of the universal simplex in the induced topology is homeomorphic to the infinite-dimensional Hilbert space ([18, 17]).

Let us now define the notion of the random simplex by analogy with the random metric space. For this purpose, define an equipped simplex as a simplex with a distinguished countable (or finite) set of extreme points dense in the whole set of extreme points. An equipped simplex has a natural continuous embedding into the space $\mathbb{R}^{\infty}$, 
and thus it is determined by the matrix whose rows are the vectors corresponding to the images of the distinguished set of extreme points. The simplex can be obtained as the closed convex hull in $\mathbb{R}^{\infty}$ of the set of these vectors. It is not difficult to describe conditions on matrices that arise in this way; they will again form a closed convex cone in the space of infinite matrices. Now it is clear how to define the random simplex: it is determined by a random matrix, or a measure in the space of matrices concentrated on the above cone. As in the case of metric spaces, one can give examples of distinguished Bernoulli-type measures.

A series of assertions similar to the main theorems of this paper is as follows:

1) The Poulsen universal simplices form an everywhere dense $G_{\delta}$-set in the set of all equipped simplices.

2) With respect to the distinguished measures, almost all simplices are universal.

3 ) The set of measures enjoying property 2 ) is an everywhere dense $G_{\delta^{-}}$set in the set of all measures on the cone with the weak topology of measures.

Thus we can justify the thesis that a random simplex is universal.

2. Random Banach spaces.

Following the same pattern, one can construct the universal $L^{1}$-type space (Gurarij space), and equipped spaces of this kind again can be determined by matrices - see [17. Random matrices will correspond to random $L^{1}$-type Banach spaces, and with respect to appropriate measures, almost every space will be universal.

3. Consider metric spaces $(X, r)$ in which the triangle inequality holds in the following strengthened form:

$$
r(x, y)^{p}+r(y, z)^{p} \geq r(x, z)^{p}, \quad p \in[1, \infty) .
$$

For $p=1$, this is the ordinary triangle inequality. In the limiting case $p=\infty$, the triangle inequality turns into the ultrametric inequality

$$
\max (r(x, y), r(y, z)) \geq r(x, z) .
$$

For brevity, spaces that satisfy the inequality with parameter $p \in[1, \infty]$ will be called p-metric.

Proposition 4 1) For all $p \in[1, \infty)$, there exists the universal $p$-metric space in the class of Polish spaces. The random (in the above sense) p-metric space is universal with probability one.

2) There is no universal ultrametric $(p=\infty)$ space.

The proof of this proposition exactly reproduces the constructions of our paper: in the same way one defines the cone of matrices, universal matrices, measures, etc. It is interesting that in the case $p=\infty$ the sets of admissible vectors (see Section 2 ) degenerate into an amoeba, which implies assertion 2). The nonexistence of the universal ultrametric space was also proved in 23. If we fix a countable set of real numbers (for example, the set of rational numbers with denominator equal to a power of some fixed simple number) and consider ultrametric spaces such that the distances 
between their points assume values only from this set, then in the class of such spaces the universal space may exist (this is the case in the example cited in the parentheses above).

At the same time, random ultrametric spaces can be defined in the same way, but most probable they will not be isometric to each other. This question will be considered elsewhere. Besides, here we do not consider examples of categories of algebraic origin: universal and random groups, algebras, lattices, etc.

4. Let us consider ordinary metric spaces but require that all distances take values in some subset $L \in \mathbb{R}$ of real numbers. For example, if this subset is $\{0,1,2\}$, then, as can be easily seen, in the set of such metric spaces there exists a universal (with respect to finite spaces) homogeneous space: this is exactly the Radó graph $\Gamma$ discussed above, regarded as a metric space (with geodesic metric); recall that any two vertices of the universal graph either are connected by an edge, or have a vertex that is connected with both of them.

Let us consider countable metric spaces with rational distances. Here there also exists a universal metric space; Urysohn's work begins exactly with the construction of this space, and its completion is the universal metric space $\mathcal{U}$. However, it seems to be an important object by itself. In this case the notion of a universal matrix is defined as above, the urysohnness criterion (see Section 3) has the same form, and one can prove the existence of the rational universal matrix in exactly the same way as above. The most important object is the group of isometries of this space. It is a closed subgroup of the group $S^{\infty}$ of all permutations of the countable set (in the weak topology), and the problem of existence of a locally finite everywhere dense subgroup of this group is of principal interest. See above for a similar question on the group of isometries of the Urysohn space.

Translated by N. V. Tsilevich.

\section{References}

[1] P. S. Urysohn. Sur un espace metrique universel. C. R. Acad. Sci. Paris 180 (1925), 803-806.

[2] P. S. Urysohn. Sur un espace metrique universel. Bull. Sci. Math. 51 (1927), $1-38$.

[3] P. S. Urysohn. On the universal metric space. In: P. S. Urysohn. Selected Works (P. S. Alexandrov, ed.), vol. 2. Nauka, Moscow, 1972, pp. 747-769.

[4] M. Gromov. Metric Structures for Riemannian and Non-Riemann ian Spaces. Birkhäuser, 1998.

[5] A. Vershik. The universal Urysohn space, Gromov's metric triples, and random metrics on the series of positive numbers. Russian Math. Surveys 53 (1998), no. 5, 921-928.

[6] A. Vershik. Random metric spaces and the universal Urysohn space. In: Fundamental Mathematics Today. MCCME, Moscow, 2003. 
[7] S. Gao, A. Kechris. On the Classification of Polish Metric Spaces up to Isometry. Memoirs of the AMS 161 (2003), no. 766.

[8] A. Vershik. Classification of measurable functions of several arguments, and invariantly distributed random matrices. Funct. Anal. Appl. 36 (2002), no. 2, 93-105.

[9] A. Vershik. Randomization of algebra and algebraization of probability. An attempt of prediction. In: "Mathematics Unlimited - 2001 and Beyond" (B. Engquist, W. Schmid, eds.), part II Springer, Berlin-Heidelberg-New York, 2001, pp. 1157-1166.

[10] A. Vershik. Dynamic theory of growth in groups: entropy, boundaries, examples. Russian Math. Surveys 55 (2000), no. 4(434), 60-128.

[11] S. Banach. Theory of Linear Operations. Moscow, 2001.

[12] M. Deza, M. Laurent. Geometry of Cuts and Metrics. Springer-Verlag, Berlin, 1997.

[13] A. Vershik. Random metric space is Urysohn space Dokl. Ross. Akad. Nauk 387 (2002), no. 6, 1-4.

[14] R. Rado. Universal graphs and universal functions. Acta Arithm. 9 (1954), 331340 .

[15] P. Erdös, A. Rényi. Asymmetric graphs. Acta Math. Acad. Sci. Hungar. 14 (1963), 295-315.

[16] P. Cameron. The random graphs. In: The Mathematics of Paul Erdös (J. Nesetril, R. L. Graham, eds.). Springer, 1996, pp. 331-351.

[17] J. Lindensrauss, G. Olsen, Y. Sterfeld. The Poulsen simplex. Ann. Inst. Fourier, Grenoble 28(1) (1978), 91-114.

[18] W. Lusku. The Gurarij spaces are unique. Arch der Mathematik 27(6) (1976), 627-635.

[19] V. V. Uspenskij. On the group of isometries of Urysohn universal metric space. Comment Math. Univ. Carolina 31 (1990), no. 1, 81-182.

[20] D. Avis. On the extreme rays of the metric cone. Canad. J. Math. 8 (1980), no. $1,126-144$.

[21] M. Katetov. On universal metric spaces. In: Proc. Sixth Prague Topological Symposium (1986) (Frolik, ed.). Helderman Verlag, Berlin, 1988, pp. 323-330.

[22] W. Sierpinski. Sur un espace metrique separable universel. Fund. Math. 33 (1945).

[23] S. A. Bogatyi. Compact homogeneity of the universal metric Urysohn space. Russian Math. Surveys 55 (2000), no. 2, 323-333.

[24] V. Pestov. Ramsay-Milman phenomenon, Urysohn space and extremally amenable groups. Israel J. Math. 127 (2002), 317-357.

[25] H. Torunczyk. Characterizing Hilbert space topology. Fund. Math. 111 (1981), no. $3,247-262$. 
[26] A. Vershik. Two lectures on the asymptotic representation theory and statistics of Young diagrams. In: Asymptotic Combinatorics with Applications to Mathematical Physics. (A. Vershik, ed.). Springer Lecture Notes in Math. 1815 (2003), pp. 161-184.

[27] F. Cucker, S. Smale. On the mathematical foundations of learning. Bull. AMS (New Ser.) 39 (2003), no. 1, 1-49.

[28] A. Kechris, V. Pestov, S. Todorcevic. Fraisse Limits, Ramsey Theory, and Topological Dynamics of Automorphism Groups. arXiv:math.LO/0305241 (2003).

[29] S. Burris. Number Theoretical Density and Logical Limit Laws. RSMM 86 AMS (2001).

[30] K. Compton. A Logical Approach to Asymptotic Combinatorics. I. First Order Properties. Ann. Math. 65 (1987), 65-96.

[31] E. Hrushovski. A new strongly minimal set. Ann. Pure Appl. Logic 62 (1993), $147-166$.

[32] B. Bolobas. Random Graphs. Academic Press, 1985.

[33] V. V. Uspenskij. The Urysohn Universal Metric Space is Homeomorphic to a Hilbert Space. arXiv:math.GN/0309101 (2003).

[34] Yu. Glebsky, L. Kogan, M. Liogon'ky, V. Talanov. Volume and fraction of satisfiability of formulas of the lower predicate calculus. Kibernetika (Kiev), no. 2 (1969), 17-27.

[35] M. Megrelishvili, L. Schroder. Globalization of Confluent Partial Actions on Topological and Metric Spaces. Preprint Elsevier Sciences (2002), 1-28.

[36] R. Fagin. Probability on finite models. Journal of Symbolic Logic, 1976.

[37] H.-D. Ebbinghaus, J. Flum. Finite Model Theory. Springer, 1999.

[38] E. Bogomolny, O. Bohigos, C. Schmit. Spectral properties of distance matrices. Preprint Univ. Paris-9, 2002.

[39] A. Vershik. Kolmogorov's example (a survey of actions of infinite-dimensional groups with invariant measure). Probab. Theory and Appl. 48 (2003), no. 2, 386391.

[40] M. Bhattacharjee, D. Macpherson. A locally finite group acting on the graph. Preprint, Univ. of Leeds. October 10, 2003.

A. Vershik

St. Petersburg Department of Steklov Institute of Mathematics vershik@pdmi.ras.ru 